\title{
Two-Dimensional Stress Intensity Factor Analysis of Cracks in Anisotropic Bimaterial
}

\author{
Chia-Huei Tu, ${ }^{1}$ Jia-Jyun Dong, ${ }^{2,3}$ Chao-Shi Chen, ${ }^{1}$ \\ Chien-Chung Ke, ${ }^{4}$ Jyun-Yong Jhan, ${ }^{1}$ and Hsien Jui $\mathbf{Y u}^{1}$ \\ ${ }^{1}$ Department of Resources Engineering, National Cheng Kung University, No. 1 University Road, Tainan City 70101, Taiwan \\ ${ }^{2}$ Graduate Institute of Applied Geology, National Central University, No. 300 Jhongda Road, Jhongli City 32001, Taiwan \\ ${ }^{3}$ Graduate Institute of Geophysics, National Central University, No. 300 Jhongda Road, Jhongli City 32001, Taiwan \\ ${ }^{4}$ Geotechnical Engineering Research Center, Sinotech Engineering Consultants, Inc., Taipei 110, Taiwan
}

Correspondence should be addressed to Chao-Shi Chen; chencs@mail.ncku.edu.tw

Received 11 June 2012; Revised 3 November 2012; Accepted 9 November 2012

Academic Editor: Giuseppe Carbone

Copyright (C) 2013 Chia-Huei Tu et al. This is an open access article distributed under the Creative Commons Attribution License, which permits unrestricted use, distribution, and reproduction in any medium, provided the original work is properly cited.

\begin{abstract}
This paper presents a 2D numerical technique based on the boundary element method (BEM) for the analysis of linear elastic fracture mechanics (LEFM) problems on stress intensity factors (SIFs) involving anisotropic bimaterials. The most outstanding feature of this analysis is that it is a singledomain method, yet it is very accurate, efficient, and versatile (i.e., the material properties of the medium can be anisotropic as well as isotropic). A computer program using the BEM formula translation (FORTRAN 90) code was developed to effectively calculate the stress intensity factors (SIFs) in an anisotropic bi-material. This BEM program has been verified and showed good accuracy compared with the previous studies. Numerical examples of stress intensity factor calculation for a straight crack with various locations in both finite and infinite bimaterials are presented. It was found that very accurate results can be obtained using the proposed method, even with relatively simple discretization. The results of the numerical analysis also show that material anisotropy can greatly affect the stress intensity factor.
\end{abstract}

\section{Introduction}

The problem of cracks between two dissimilar materials has been widely studied over the past several decades, stemming mainly from the desire to understand the failure modes of composites, including structures, rocks, and concrete. Williams [1] presented the first study of the plane problem of cracks between dissimilar isotropic materials. Williams showed that stresses possess the singularity of $r^{-1 / 2 \pm i \varepsilon}$, where $r$ is the radius distance from the crack tip and $\varepsilon$ is a bi-material constant. England [2] investigated the problem of finite cracks between dissimilar isotropic materials. Rice and Sih [3] studied similar problems and derived the expressions of the stress fields near crack tips. Rice [4] reexamined the elastic fracture mechanics concepts of the isotropic interfacial crack and introduced an intrinsic material length scale so that the definition of the stress intensity factors (SIFs) possessed the same physical significance as those for homogeneous cracks.
Clements [5] and Willis [6] extended the problem studied by England [2] for dissimilar anisotropic materials. They also showed the oscillatory behavior of the stresses and the phenomenon of interpenetration of the crack face near the crack tip in anisotropic interface cracks. Wu [7] extended the problem studied by Rice [4] for anisotropic bi-material cracks. Recently, many authors [7-10] have conducted studies on interfacial cracks in anisotropic materials and different definitions for the stress intensity factor existence. However, the data on different crack locations, crack lengths, and degree of material anisotropy are scarce in the literature.

Recently, several BEMs have been proposed for the study of cracked media [11-13]. It involves two sets of boundary integral equations and is, in general, superior to the aforementioned BEMs. Consequently, general mixed mode crack problems can be solved in a BEM formulation. The singledomain analysis can eliminate remeshing problems, which are typical of the FEM and the subregional BEM. 
In this paper, we develop a technique for single-domain BEM formulation in which neither the artificial boundary nor the discretization along the uncracked interface is necessary. Chen et al. [14] presented this single-domain BEM formulation for homogeneous materials. We combined it with Green's functions of bi-materials [15] to extend it to anisotropic bi-materials. The BEM formulation is such that the displacement integral equation is applied only to the outer boundary, and the traction integral equation is applied only to one side of the cracked surface. A decoupling technique can be used to determine mixed mode SIFs based on the relative displacement at the crack tip. Numerical cases for mixed mode SIFs for anisotropic bi-materials with different crack locations and degrees of material anisotropy are presented. The numerical results obtained using the BEM formulation are verified by several previous studies $[8,16-18]$.

\section{Methodology}

2.1. Basic Equations for Anisotropic Elasticity. For linear elastic, homogeneous, and anisotropic material, the stress and displacement fields can be formulated in terms of two analytical functions, $\phi_{k}\left(z_{k}\right)$ of the complex variables $z_{k}=$ $x+\mu_{k} y(k=1,2)$ where $\mu_{k}$ is the root of the following characteristic equation [19]:

$$
a_{11} \mu^{4}-2 a_{16} \mu^{3}+\left(2 a_{12}+a_{66}\right) \mu^{2}-2 a_{26} \mu+a_{22}=0,
$$

where the coefficient $a_{i j}$ is the compliance component calculated using the $x-y$ coordinate system. The detailed relationships of these components with material elasticity can be found in Chen et al. [14]. If the root $\mu_{j}$ of (1) is assumed to be distinct, the general expressions for the stress and displacements are

$$
\begin{gathered}
\sigma_{x}=2 \operatorname{Re}\left[\mu_{1}^{2} \phi_{1}^{\prime}\left(z_{1}\right)+\mu_{2}^{2} \phi_{2}^{\prime}\left(z_{2}\right)\right], \\
\sigma_{y}=2 \operatorname{Re}\left[\phi_{1}^{\prime}\left(z_{1}\right)+\phi_{2}^{\prime}\left(z_{2}\right)\right], \\
\tau_{x y}=-2 \operatorname{Re}\left[\mu_{1} \phi_{1}^{\prime}\left(z_{1}\right)+\mu_{2} \phi_{2}^{\prime}\left(z_{2}\right)\right], \\
u=2 \operatorname{Re}\left[A_{11} \phi_{1}\left(z_{1}\right)+A_{12} \phi_{2}\left(z_{2}\right)\right], \\
v=2 \operatorname{Re}\left[A_{21} \phi_{1}\left(z_{1}\right)+A_{22} \phi_{2}\left(z_{2}\right)\right],
\end{gathered}
$$

where the elements of the complex matrices $A$ are

$$
\begin{gathered}
A_{1 j}=a_{11} \mu_{j}^{2}+a_{22}-a_{16} \mu_{j}, \\
A_{2 j}=a_{12} \mu_{j}+\frac{a_{22}}{\mu_{j}}-a_{26} \quad(j=1,2) .
\end{gathered}
$$

The traction components in the $x$ and $y$ directions are

$$
\begin{aligned}
& T_{x}=2 \operatorname{Re}\left[\mu_{1} \phi_{1}\left(z_{1}\right)+\mu_{2} \phi_{2}\left(z_{2}\right)\right], \\
& T_{y}=-2 \operatorname{Re}\left[\phi_{1}\left(z_{1}\right)+\phi_{2}\left(z_{2}\right)\right] .
\end{aligned}
$$

Here, the complex analytical functions $\phi_{i}\left(z_{i}\right)$ can in general express (2) and (4) as follows [19-21]:

$$
\begin{gathered}
u_{i}=2 \operatorname{Re}\left[A_{1 i} \phi_{1}\left(z_{1}\right)+A_{2 i} \phi_{2}\left(z_{2}\right)\right], \\
T_{i}=-2 \operatorname{Re}\left[B_{1 i} \phi_{1}\left(z_{1}\right)+B_{2 i} \phi_{2}\left(z_{2}\right)\right], \\
\sigma_{2 i}=2 \operatorname{Re}\left[B_{1 i} \phi_{1}^{\prime}\left(z_{1}\right)+B_{2 i} \phi_{2}^{\prime}\left(z_{2}\right)\right], \\
\sigma_{1 i}=-2 \operatorname{Re}\left[B_{1 i} \phi_{1}^{\prime}\left(z_{1}\right)+B_{2 i} \phi_{2}^{\prime}\left(z_{2}\right)\right], \quad(i=1,2),
\end{gathered}
$$

where $z_{j}=x+\mu_{j} y$; Re denotes the real part of a complex variable or function; a prime denotes the derivative; the complex number $\mu_{j}(j=1,2)$; the elements of complex matrices $A$ are defined in (3); and the elements of complex matrices $B$ can be defined as

$$
B_{i j}=\left[\begin{array}{cc}
-\mu_{1} & -\mu_{2} \\
1 & 1
\end{array}\right]
$$

Assume that the medium is composed of two joined dissimilar anisotropic and elastic half-planes. Let the interface be along the $x$-axis and let the upper $(y>0)$ and lower $(y<0)$ half-planes be occupied by materials \#1 and \#2, respectively (as shown in Figure 1).

Considering a concentrated force acting at the source point $\left(x^{0}, y^{0}\right)$ in material $\# 2\left(y^{0}<0\right)$, we express the complex vector function as [20]

$$
\phi(z)= \begin{cases}\phi^{\# 1}(z), & z \in(1), \\ \phi^{\# 2}(z)+\phi_{\# 2}^{0}(z), & z \in(2),\end{cases}
$$

where the vector function is

$$
\phi(z)=\left[\phi_{1}(z), \phi_{2}(z)\right]^{T}
$$

where the argument has the generic form $z=x+\mu y$.

In (7), $\phi_{\# 2}^{0}$ is a singular solution corresponding to a point force acting at the point $\left(x^{0}, y^{0}\right)$ on an anisotropic infinite plane with the elastic properties of material \#2. This singular solution can be expressed as $[20,21]$

$$
\phi_{k}^{0}\left(z_{k}\right)=-\frac{1}{2 \pi}\left[H_{k 1} P_{1} \ln \left(z_{k}-s_{k}\right)+H_{k 2} P_{2} \ln \left(z_{k}-s_{k}\right)\right],
$$

where $s_{k}=x^{0}+\mu_{k} y^{0}, P_{k}$ is the magnitude of the point force in the $k$-direction, and

$$
H=A^{-1}\left(Y^{-1}+\bar{Y}^{-1}\right)^{-1}, \quad Y=i A B^{-1}
$$

where $i=\sqrt{-1}$, the overbar indicates the complex conjugate, and superscript -1 indicates the matrix inverse. Two unknown vector functions, $\phi^{\# 1}(z)$ and $\phi^{\# 2}(z)$, are solved in (7). The former is analytic in material \#1, and the latter is analytic in material \#2. These expressions can be found by requiring the continuity of the resultant traction and displacement 


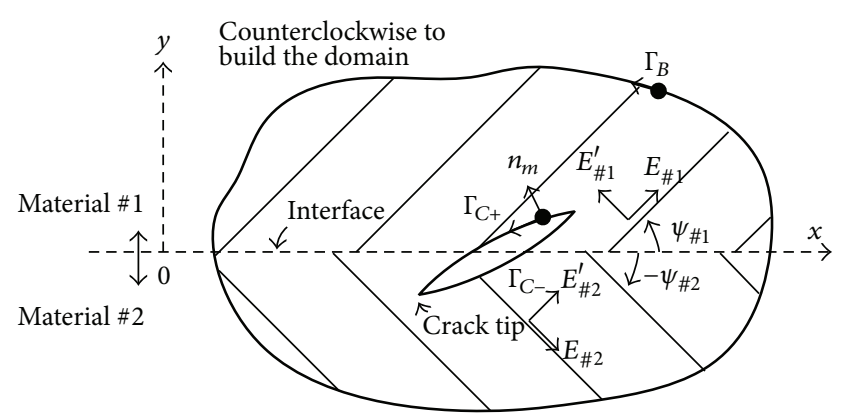

$\Gamma_{B}$ Displacement equation $\Gamma_{C}$ Traction equation

FIGURE 1: Definition of the coordinate systems in an anisotropic bimaterial.

across the interface in addition to the standard analytic continuation arguments. Substituting (9) for (7), we obtain

$$
\begin{array}{r}
\phi^{\# 1}(Z)=B_{\# 1}^{-1}\left(Y_{\# 1}+\bar{Y}_{\# 2}\right)^{-1}\left(\bar{Y}_{\# 2}+Y_{\# 2}\right) B_{\# 2} \phi_{\# 2}^{0}(z), \\
z \in \# 1, \\
\phi^{\# 2}(Z)=B_{\# 2}^{-1}\left(\bar{Y}_{\# 1}+Y_{\# 2}\right)^{-1}\left(\bar{Y}_{\# 2}-\bar{Y}_{\# 1}\right) \bar{B}_{\# 2} \phi_{\# 2}^{0}(z), \\
z \in \# 2 .
\end{array}
$$

Therefore, the complex vector functions can be expressed as

$$
\phi(z)=\left\{\begin{array}{cc}
B_{\# 1}^{-1}\left(Y_{\# 1}+\bar{Y}_{\# 2}\right)^{-1} & \\
\times\left(\bar{Y}_{\# 2}+Y_{\# 2}\right) B_{\# 2} \phi_{\# 2}^{0}(z), & z \in \# 1, \\
B_{\# 2}^{-1}\left(\bar{Y}_{\# 1}+Y_{\# 2}\right)^{-1}\left(\bar{Y}_{\# 2}-\bar{Y}_{\# 1}\right) & \\
\times \bar{B}_{\# 2} \phi_{\# 2}^{0}(z)+\phi_{\# 2}^{0}(z), & z \in \# 2 .
\end{array}\right.
$$

In (12), subscripts \#1 and \#2 are used to denote that the corresponding matrix or vector is for material \#1 $(y>0)$ and material \#2 $(y<0)$, respectively.

Similarly, for a point force in material \#1 $\left(y^{0}>0\right)$, the complex vector functions can be expressed as

$$
\phi(z)=\left\{\begin{array}{cc}
B_{\# 1}^{-1}\left(\bar{Y}_{\# 2}+Y_{\# 1}\right)^{-1} & \\
\times\left(\bar{Y}_{\# 1}-\bar{Y}_{\# 2}\right) \bar{B}_{\# 1} \bar{\phi}_{\# 2}^{0}(z)+\phi_{\# 1}^{0}(z), & z \in \# 1, \\
B_{\# 2}^{-1}\left(Y_{\# 2}+\bar{Y}_{\# 1}\right)^{-1} & \\
\times\left(\bar{Y}_{\# 1}+Y_{\# 1}\right) B_{\# 1} \phi_{\# 1}^{0}(z), & z \in \# 2,
\end{array}\right.
$$

where vector function $\phi_{\# 1}^{0}$ is the infinite-plane solution given in (9), but with the elastic properties of material \#1.

The Green's functions of the displacement and traction can be obtained by substituting the complex vector functions in (12) and (13) for (5). Here, $U_{k l}^{*}$ is the Green's function for displacement and $T_{k l}^{*}$ is the Green's function for traction. Superscripts and subscripts $\# 1$ and $\# 2$ are used to denote the corresponding quantities for materials \#1 $(y>0)$ and $\# 2(y<0)$, respectively [15]. (i) For source point $(s)$ and field point $(z)$ on material $\# 1(y>0)$,

$$
\begin{aligned}
& U_{k l}^{*}=\frac{-1}{\pi} \operatorname{Re}\left\{\sum_{j=1}^{2} A_{l j}^{\# 1}[\right. \ln \left(z_{j}^{\# 1}-s_{j}^{\# 1}\right) H_{j k}^{\# 1} \\
&\left.\left.+\sum_{i=1}^{2} W_{j i}^{11} \ln \left(z_{j}^{\# 1}-s_{i}^{\# 1}\right) \overline{H_{i k}^{\# 1}}\right]\right\}, \\
& T_{k l}^{*}=\frac{1}{\pi} \operatorname{Re}\left\{\sum _ { j = 1 } ^ { 2 } B _ { l j } ^ { \# 1 } \left[\frac{\mu_{j}^{\# 1} n_{x}-n_{y}}{z_{j}^{\# 1}-s_{j}^{\# 1}} H_{j k}^{\# 1}\right.\right. \\
&\left.\left.+\sum_{i=1}^{2} W_{j i}^{11} \frac{\mu_{j}^{\# 1} n_{x}-n_{y}}{z_{j}^{\# 1}-s_{i}^{\# 1}} \overline{H_{i k}^{\# 1}}\right]\right\},
\end{aligned}
$$

where the matrix $H$ is defined in (10) with the anisotropic elastic properties of material \#1, and

$$
W^{11}=B_{\# 1}^{-1}\left(Y_{\# 1}+\bar{Y}_{\# 2}\right)^{-1}\left(\bar{Y}_{\# 1}-\bar{Y}_{\# 2}\right) \bar{B}_{\# 1} \text {. }
$$

(ii) For source point $(s)$ and field point $(z)$ on material $\# 1(y>0)$ and field point $(z)$ on material \#2 $(y<0)$,

$$
\begin{gathered}
U_{k l}^{*}=\frac{-1}{\pi} \operatorname{Re}\left\{\sum_{j=1}^{2} A_{l j}^{\# 2}\left[\sum_{i=1}^{2} W_{j i}^{12} \ln \left(z_{j}^{\# 2}-s_{i}^{\# 1}\right) H_{i k}^{\# 1}\right]\right\}, \\
T_{k l}^{*}=\frac{1}{\pi} \operatorname{Re}\left\{\sum_{j=1}^{2} B_{l j}^{\# 2}\left[\sum_{i=1}^{2} W_{j i}^{12} \frac{\mu_{j}^{\# 2} n_{x}-n_{y}}{z_{j}^{\# 2}-s_{i}^{\# 1}} \bar{H}_{i k}^{\# 1}\right]\right\},
\end{gathered}
$$

with

$$
W^{12}=B_{\# 2}^{-1}\left(Y_{\# 2}+\bar{Y}_{\# 1}\right)^{-1}\left(\bar{Y}_{\# 1}-Y_{\# 1}\right) B_{\# 1} .
$$

(iii) For source point $(s)$ and field point $(z)$ on material $\# 2(y<0)$,

$$
\begin{aligned}
& U_{k l}^{*}=\frac{-1}{\pi} \operatorname{Re}\left\{\sum_{j=1}^{2} A_{l j}^{\# 2}[\right. \ln \left(z_{j}^{\# 2}-s_{j}^{\# 2}\right) H_{j k}^{\# 2} \\
&\left.\left.+\sum_{i=1}^{2} W_{j i}^{22} \ln \left(z_{j}^{\# 2}-s_{i}^{\# 2}\right) \bar{H}_{i k}^{\# 2}\right]\right\}, \\
& T_{k l}^{*}=\frac{1}{\pi} \operatorname{Re}\left\{\sum _ { j = 1 } ^ { 2 } B _ { l j } ^ { \# 2 } \left[\frac{\mu_{j}^{\# 2} n_{x}-n_{y}}{z_{j}^{\# 2}-s_{j}^{\# 2}} H_{j k}^{\# 2}\right.\right. \\
&\left.\left.+\sum_{i=1}^{2} W_{j i}^{22} \frac{\mu_{j}^{\# 2} n_{x}-n_{y}}{z_{j}^{\# 2}-s_{i}^{\# 2}} \bar{H}_{i k}^{\# 2}\right]\right\},
\end{aligned}
$$

where matrix $H$ is defined in (10) with the anisotropic elastic properties of material $\# 2$, and

$$
W^{22}=B_{\# 2}^{-1}\left(Y_{\# 2}+\bar{Y}_{\# 1}\right)^{-1}\left(\bar{Y}_{\# 2}-\bar{Y}_{\# 1}\right) \bar{B}_{\# 2} \text {. }
$$


(iv) For source point $(s)$ and field point $(z)$ on materials $\# 2(y<0)$ and field point $(z)$ on material \#1 $(y>0)$,

$$
\begin{gathered}
U_{k l}^{*}=\frac{-1}{\pi} \operatorname{Re}\left\{\sum_{j=1}^{2} A_{l j}^{\# 1}\left[\sum_{i=1}^{2} W_{j i}^{21} \ln \left(z_{j}^{\# 1}-s_{i}^{\# 2}\right) H_{i k}^{\# 2}\right]\right\}, \\
T_{k l}^{*}=\frac{1}{\pi} \operatorname{Re}\left\{\sum_{j=1}^{2} B_{l j}^{\# 1}\left[\sum_{i=1}^{2} W_{j i}^{21} \frac{\mu_{j}^{\# 1} n_{x}-n_{y}}{z_{j}^{\# 1}-s_{i}^{\# 2}} \bar{H}_{i k}^{\# 2}\right]\right\},
\end{gathered}
$$

with

$$
W^{21}=B_{\# 1}^{-1}\left(Y_{\# 1}+\bar{Y}_{\# 2}\right)^{-1}\left(\bar{Y}_{\# 2}-Y_{\# 2}\right) B_{\# 2} \text {. }
$$

It should be noted that these Green's functions can be used to solve both plane stress and plane strain problems for anisotropic bi-materials. Although the isotropic solution cannot be analytically reduced from these Green's functions, one can numerically approximate it by selecting a very weak anisotropic (or nearly isotropic) medium $[22,23]$.

2.2. Single-Domain Boundary Integral Equations. In this section, we present single-domain boundary integral equations in which neither the artificial boundary nor the discretization along the uncracked interface is necessary. This singledomain boundary integral equation was used recently by Chen et al. [14] for homogeneous materials, and it is now extended to anisotropic bi-materials.

For a point $s_{k, B}^{0}$ on the uncracked boundary, the displacement integral equation applied to the outer boundary results in the following form $\left(s_{k, B}^{0} \in \Gamma_{B}\right.$ only, as shown in Figure 1):

$$
\begin{aligned}
b_{i j}\left(s_{k, B}^{0}\right) & u_{j}\left(s_{k, B}^{0}\right) \\
& +\int_{\Gamma_{B}} T_{i j}^{*}\left(z_{k, B}, s_{k, B}^{0}\right) u_{j}\left(z_{k, B}\right) d \Gamma\left(z_{k, B}\right) \\
& +\int_{\Gamma_{C}} T_{i j}^{*}\left(z_{k, C}, s_{k, B}^{0}\right)\left[u_{j}\left(z_{k, C+}\right)-u_{j}\left(z_{k, C-}\right)\right] d \Gamma\left(z_{k, C}\right) \\
= & \int_{\Gamma_{B}} U_{i j}^{*}\left(z_{k, B}, s_{k, B}^{0}\right) t_{j}\left(z_{k, B}\right) d \Gamma\left(z_{k, B}\right),
\end{aligned}
$$

where $i, j, k=1,2 ; T_{i j}^{*}$ and $U_{i j}^{*}$ are the Green's tractions and displacements given in (14), (16), (18), and (20); $u_{j}$ and $t_{j}$ are the boundary displacements and tractions, respectively; and $z_{k}$ and $s_{k, B}^{0}$ are the field points and the source points on boundary $\Gamma$ of the domain, respectively. $\Gamma_{C}$ has the same outward normal as $\Gamma_{C_{+}} \cdot b_{i j}$ are coefficients that depend only on the local geometry of the uncracked boundary at point $s_{k, B}^{0}$ and are equal to $\delta_{i j} / 2$ for a smooth boundary. Here, subscripts $B$ and $C$ denote the outer boundary and the cracked surface, respectively. In deriving (22), we have assumed that the tractions on the two faces of a crack are equal and opposite. We emphasize here that since the bi-material Green's functions are included in (22), discretization along the interface can be avoided, with the exception of the interfacial crack part, which is treated by the traction integral equation presented in the following.

It should be noted that all the terms on the right-hand side of (22) have weak singularities and thus are integrable. Although the second term on the left-hand side of (22) has a strong singularity, it can be treated using the rigid-body motion method.

The traction integral equation (for $s_{k}^{0}$ being a smooth point on the crack) applied to one side of the cracked surface is $\left(s_{k, C}^{0} \in \Gamma_{C+}\right.$ only)

$$
\begin{gathered}
0.5 t_{j}\left(s_{k, C}^{0}\right)+n_{m}\left(s_{k, C}^{0}\right) \int_{\Gamma \mathrm{B}} C_{\lim k} T_{i j, k}^{*}\left(s_{k, C}^{0}, z_{k, B}\right) \\
\times u_{j}\left(z_{k, B}\right) d \Gamma\left(z_{k, B}\right) \\
+n_{m}\left(s_{k, C}^{0}\right) \int_{\Gamma C} C_{\lim k} T_{i j, k}^{*}\left(s_{k, C}^{0}, z_{k, C}\right) \\
\times\left[u_{j}\left(z_{k, C+}\right)-u_{j}\left(z_{k, C-}\right)\right] d \Gamma\left(z_{k, C}\right) \\
=n_{m}\left(s_{k, C}^{0}\right) \int_{\Gamma B} C_{\lim k} U_{i j, k}^{*}\left(s_{k, C}^{0}, z_{k, B}\right) t_{j}\left(z_{k, B}\right) d \Gamma\left(z_{k, B}\right),
\end{gathered}
$$

where $n_{m}$ is the unit outward normal at cracked surface $s_{k, C}^{0}$ and $C_{\lim k}$ is the fourth-order stiffness tensor.

Equations (22) and (23) form a pair of boundary integral equations $[14,21,24]$ and can be used to calculate SIFs in anisotropic bi-materials. The main feature of the BEM formulation is that it is a single-domain formulation, with the displacement integral equation (22) being collocated only on the uncracked boundary and the traction integral equation (23) only on one side of the crack surface. For problems without cracks, one only needs (22), with the integral on the cracked surface being discarded. Equation (22) then reduces to the well-known displacement integral on the uncracked boundary.

The internal stresses $\sigma\left(z_{k}\right)$ are determined using the following expression:

$$
\begin{array}{r}
\sigma_{\operatorname{lm}}\left(z_{k}\right)+\int_{\Gamma \mathrm{B}} C_{\lim k} T_{i j, k}^{*}\left(s_{k, C}^{0}, z_{k, B}\right) u_{j}\left(z_{k, B}\right) d \Gamma\left(z_{k, B}\right) \\
+\int_{\Gamma C} C_{\lim k} T_{i j, k}^{*}\left(s_{k, C}^{0}, z_{k, C}\right) \\
\times\left[u_{j}\left(z_{k, C+}\right)-u_{j}\left(z_{k, C-}\right)\right] d \Gamma\left(z_{k, C}\right) \\
=\int_{\Gamma B} C_{\lim k} U_{i j, k}^{*}\left(s_{k, C}^{0}, z_{k, B}\right) t_{j}\left(z_{k, B}\right) d \Gamma\left(z_{k, B}\right) .
\end{array}
$$

For given solutions (related to the body force of gravity, rotational forces, and the far-field stresses), the boundary integral equations (22) and (23) can be discretized and solved numerically for the unknown boundary displacements (or displacement discontinuities on the cracked surface) and tractions. In solving these equations, the hypersingular integral term in (23) can be handled using an accurate and 
efficient Gauss quadrature formula, which is similar to the traditional weighted Gauss quadrature but has a different weight [25].

2.3. Evaluation of Stress Intensity Factor. In fracture mechanics analysis, especially in the calculation of SIFs, one needs to know the asymptotic behavior of the displacements and stresses near the crack tip. In our BEM analysis of SIFs, we propose to use the extrapolation method of cracktip displacement. We, therefore, need to know the exact asymptotic behavior of the relative crack displacement (RCD) behind the crack tip. The form of the asymptotic expression depends on the location of the crack tip. In this study, two cases are discussed: a crack tip in homogeneous material and an interfacial crack tip.

(i) Crack Tip in Homogeneous Material. Assume that the crack tip is in material \#1. The asymptotic behavior of the relative displacement at a distance $r$ behind the crack tip can be expressed in terms of the three SIFs as [17]

$$
\Delta u(r)=2 \sqrt{\frac{2 r}{\pi}} \operatorname{Re}\left(Y_{\# 1}\right) K,
$$

where $K=\left[K_{\mathrm{II}}, K_{\mathrm{I}}, K_{\mathrm{III}}\right]^{T}$ is the SIF vector and $Y$ is a matrix with elements related to the anisotropic properties in material \#1, as defined in (10).

In order to calculate the square-root characteristic of the RCD near the crack tip, we constructed the following new crack tip element with the tip at $\xi=-1$ (as shown in Figure 2(e)):

$$
\Delta u_{i}=\sum_{k=1}^{3} f_{k} \Delta u_{i}^{k}
$$

where subscript $i$ denotes the RCD component and superscript $k(1,2,3)$ denotes the RCDs at nodes $\xi=-2 / 3,0,2 / 3$ (as shown in Figure 2(b)), respectively. The shape functions $f_{k}$ are those introduced by [25]

$$
\begin{aligned}
& f_{1}=\frac{3 \sqrt{3}}{8} \sqrt{\xi+1}\left[5-8(\xi+1)+3(\xi+1)^{2}\right], \\
& f_{2}=\frac{1}{4} \sqrt{\xi+1}\left[-5+18(\xi+1)+9(\xi+1)^{2}\right], \\
& f_{3}=\frac{3 \sqrt{3}}{8 \sqrt{5}} \sqrt{\xi+1}\left[1-4(\xi+1)+3(\xi+1)^{2}\right] .
\end{aligned}
$$

In this case, the relation of the RCDs at a distance $r$ behind the crack tip and the SIFs can be found as [26-28]

$$
\begin{aligned}
& \Delta u_{1}=2 \sqrt{\frac{2 r}{\pi}}\left(H_{11} K_{\mathrm{I}}+H_{12} K_{\mathrm{II}}\right), \\
& \Delta u_{2}=2 \sqrt{\frac{2 r}{\pi}}\left(H_{21} K_{\mathrm{I}}+H_{22} K_{\mathrm{II}}\right),
\end{aligned}
$$

where

$$
\begin{aligned}
& H_{11}=\operatorname{Im}\left(\frac{\mu_{2} P_{11}-\mu_{2} P_{12}}{\mu_{1}-\mu_{2}}\right), \\
& H_{12}=\operatorname{Im}\left(\frac{P_{11}-P_{12}}{\mu_{1}-\mu_{2}}\right),
\end{aligned}
$$

$$
\begin{aligned}
& H_{21}=\operatorname{Im}\left(\frac{\mu_{2} P_{21}-\mu_{2} P_{22}}{\mu_{1}-\mu_{2}}\right), \\
& H_{22}=\operatorname{Im}\left(\frac{P_{21}-P_{22}}{\mu_{1}-\mu_{2}}\right) .
\end{aligned}
$$

Substituting the RCDs into (26) and (28), we may obtain a set of algebraic equations with which the SIFs $K_{\mathrm{I}}$ and $K_{\mathrm{II}}$ can be solved.

(ii) Interfacial Crack Tip. In this case, the relative crack displacements at a distance $r$ behind the interfacial crack tip can be expressed in terms of the three SIFs as [29]

$$
\Delta u(r)=\left(\sum_{j=1}^{3} c_{j} D Q_{j} e^{-\pi \delta_{j}} r^{(1 / 2)+\delta_{j}}\right) K,
$$

where $c_{j}, \delta_{j}, Q_{j}$, and $D$ are the relative parameters in materials $\# 1$ and $\# 2$. Utilizing (10), we defined the matrix of a material as

$$
Y_{\# 1}+\bar{Y}_{\# 2}=D-i V \text {, }
$$

where $D$ and $V$ are two real matrices. These two matrices are used to define matrix $P$ as

$$
P=-D^{-1} V
$$

The characteristic $\beta$ relative to material is as follows:

$$
\beta=\sqrt{-\frac{1}{2} \operatorname{tr}\left(P^{2}\right)} .
$$

We use the characteristic $\beta$ to define oscillation index $\varepsilon$ as

$$
\begin{gathered}
\varepsilon=\frac{1}{2 \pi} \ln \frac{1+\beta}{1-\beta}=\frac{1}{\pi} \tanh ^{-1} \beta, \\
\delta_{1}=0, \quad \delta_{2}=\varepsilon, \quad \delta_{3}=-\varepsilon, \\
Q_{1}=P^{2}+\beta^{2} I, \quad Q_{2}=P(P-i \beta I), \\
Q_{3}=P(P+i \beta I),
\end{gathered}
$$

where $I$ is a $3 \times 3$ identity matrix.

The relationship between characteristic $\beta$ and oscillation index $\varepsilon$ is used to define constant $c_{j}$ as

$$
\begin{gathered}
c_{1}=\frac{2}{\sqrt{2 \pi} \beta^{2}}, \\
c_{2}=\frac{-e^{-\pi \varepsilon} d^{i \varepsilon}}{\sqrt{2 \pi}(1+2 i \varepsilon) \beta^{2} \cosh (\pi \varepsilon)}, \\
c_{3}=\frac{-e^{-\pi \varepsilon} d^{i \varepsilon}}{\sqrt{2 \pi}(1-2 i \varepsilon) \beta^{2} \cosh (\pi \varepsilon)},
\end{gathered}
$$

where $d$ is the characteristic distance along the material interface to the crack tip. 


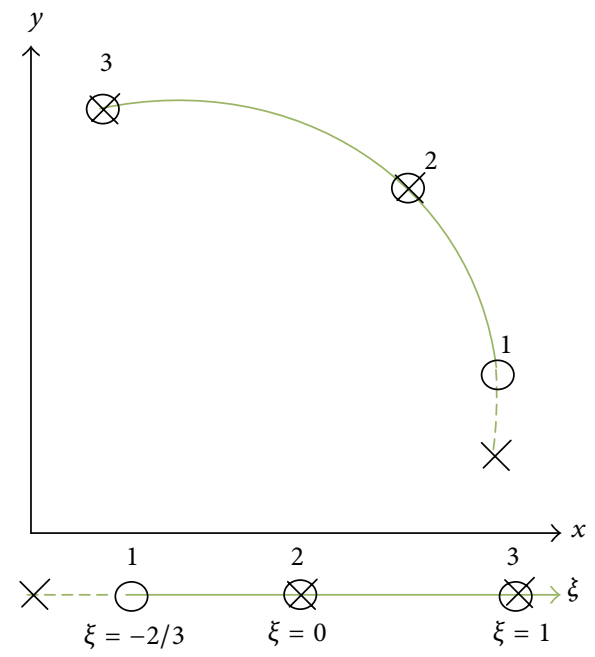

(a) Discontinuous quadratic element of Type I

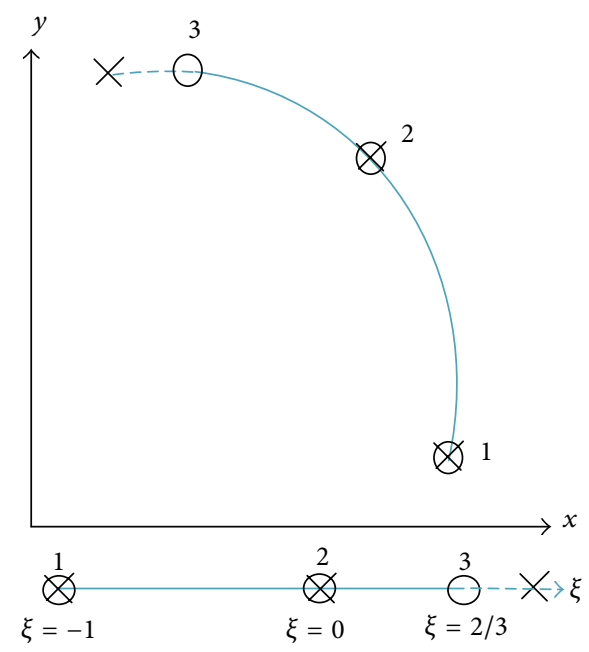

(c) Discontinuous quadratic element of Type III

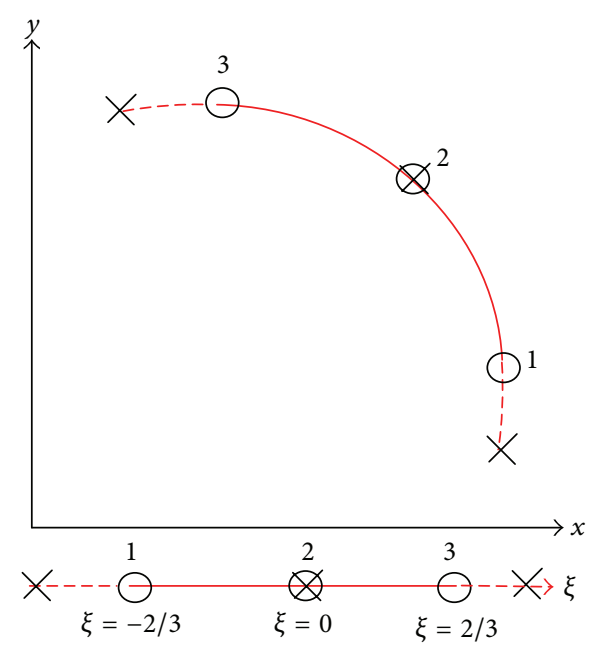

(b) Crack surface quadratic element of Type II

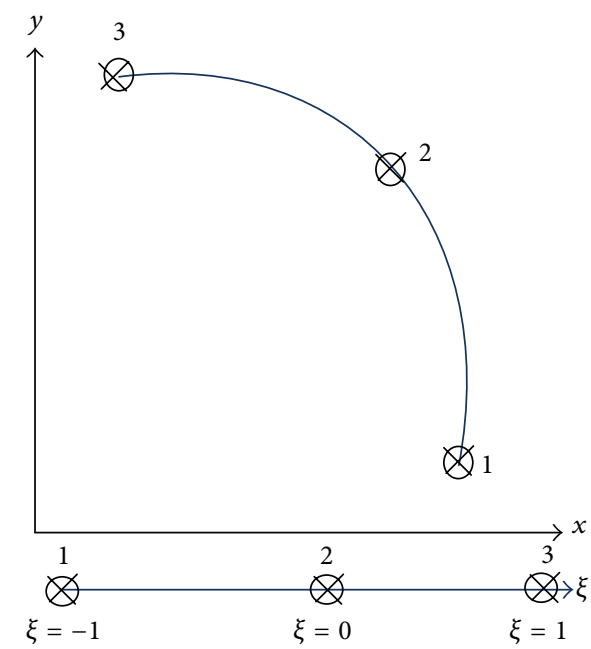

(d) Discontinuous quadratic element of Type IV

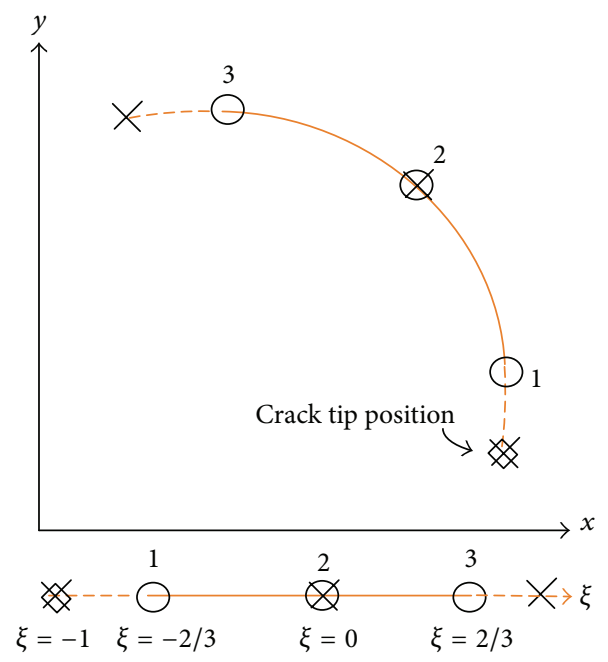

(e) Crack tip quadratic element of Type V

FIGURE 2: Three-geometric-node quadratic elements used to approximate the uncracked boundary and the crack for two-dimensional problems. 
Comparing (25) with (30), we noticed that while the relative crack displacement behaves as a square root for a crack tip in a homogeneous medium, an interfacial crack-tip's behavior is $r^{(1 / 2)+i \delta}$, a square root feature multiplied by a weak oscillation.

Equation (30) can be written in the following form, which is more convenient for the current numerical applications:

$$
\Delta u(r)=\sqrt{\frac{2 r}{\pi}} M\left(\frac{r}{d}\right) K
$$

where $d$ is the characteristic length and $M$ is a matrix function expressed as

$$
\begin{aligned}
M(x)=\frac{D}{\beta^{2}}\{ & \left(P^{2}+\beta^{2} I\right) \\
- & \left([\cos (\varepsilon \ln x)+2 \varepsilon \sin (\varepsilon \ln x)] P^{2}\right. \\
& +\beta[\sin (\varepsilon \ln x)-2 \varepsilon \cos (\varepsilon \ln x)] P) \\
& \left.\times\left(\left(1+4 \varepsilon^{2}\right) \cosh (\pi \varepsilon)\right)^{-1}\right\} .
\end{aligned}
$$

Again, in order to capture the square root and weak oscillatory behavior, we constructed a crack-tip element with the tip at $\xi=-1$ (as shown in Figure 2(e)) in terms of which the relative crack displacement is expressed as

$$
\Delta u(r)=M\left(\frac{r}{d}\right)\left[\begin{array}{lll}
f_{1} \Delta u_{1}^{1} & f_{2} \Delta u_{1}^{2} & f_{3} \Delta u_{1}^{3} \\
f_{1} \Delta u_{2}^{1} & f_{2} \Delta u_{2}^{2} & f_{3} \Delta u_{2}^{3} \\
f_{1} \Delta u_{3}^{1} & f_{2} \Delta u_{3}^{1} & f_{3} \Delta u_{3}^{3}
\end{array}\right]
$$

2.4. Construction of the Numerical Model. Quadratic elements are often used to model problems with curvilinear geometry. Three-geometric-node quadratic elements can be subdivided into five types, as shown in Figure 2, during analysis. For these elements, both the geometry and the boundary quantities are approximated by intrinsic coordinate $\xi$, and shape function $f_{k}$ is obtained from (27). Generally, the three geometric nodes of an element are used as collocation points. Such an element is referred to as a continuous quadratic element of Type IV (as shown in Figure 2(d)). To model the corner points or the points where there is a change in the boundary conditions, one-sided discontinuous quadratic elements (commonly referred to as partially discontinuous elements) are used. These elements can be one of four types depending on which extreme collocation point has been shifted inside the element to model the geometric or physical singularity. If the third collocation node is shifted inside, then the resulting element is referred to as a partially discontinuous quadratic element of Type I (as shown in Figure 2(a)). If the first node is shifted inside, then the element is called a partially discontinuous quadratic element of Type III (as shown in Figure 2(c)). One may opt for moving both extreme nodes inside, which results in discontinuous elements of Type II and Type V (to model the cracked surface or the points where there is in the crack tip positions) as shown in Figures 2(b) and 2(e), respectively. In the graphical representation of these elements, we use an " $x$ " for a geometric node, a " $\bigcirc$ " for a collocation node, and a " $\nabla$ " for the crack tip position.

\section{Verification of the BEM Program}

The Green's functions and the particular crack-tip elements were incorporated into the boundary integral equations, and the results were programmed using FORTRAN code. In this section, the following numerical examples are presented to verify the formulation and to show the efficiency and versatility of the present BEM method for problems related to fractures in bi-material.

3.1. Horizontal Crack in Material \#1. A horizontal crack under uniform pressure $P$ is shown in Figure 3. The crack, whose length is $2 a$, is located distance $d$ from the interface. The Poisson's ratios for materials \#1 and \#2 are $v_{\# 1}=v_{\# 2}=$ 0.3 , and the ratio of the shear modulus $G_{\# 2} / G_{\# 1}$ varies. A plane stress condition is assumed. In order to calculate the SIFs at crack tip A or B, 20 quadratic elements were used to discretize the crack surface. The results are given in Table 1 for various values of the shear modulus ratio. They are compared to the results of Isida and Noguchi [16], who used a body force integral equation method, and those of Ryoji and Sang-Bong [17], who used a multidomain BEM formulation. It can be seen in this table that the results are in agreement.

3.2. Interfacial Crack in an Anisotropic Bi-Material Plate. The following example is used for comparison with results from the literature in order to demonstrate the accuracy of the BEM approach for an interfacial crack in an anisotropic bimaterial plate. The geometry is that of a rectangular plate, as shown in Figure 4. For the comparison, the crack length is $2 a, h=2 w, a / w=0.4$, and static tensile loading $\sigma$ is applied to the upper and the lower boundaries of the plate.

A plane stress condition is assumed. The anisotropic elastic properties for materials \#1 and \#2 are given in Table 2. The normalized complex stress intensity factors at crack tips A and B are listed in Table 3 together with those from Sang-Bong et al. [18], who used a multidomain BEM formulation, and the results from Wünsche et al. [8] for a finite body. The outer boundary and interfacial crack surface were discretized with 20 continuous and 20 discontinuous quadratic elements, respectively. Table 3 shows that the BEM approach produces results that agree well with those obtained by other researchers.

\section{Numerical Analysis}

After its accuracy was checked, the BEM program was applied to more challenging problems involving an anisotropic bimaterial to determine the SIFs of crack tips. A generalized plane stress condition was assumed in all the problems. In the analysis, zero traction on the crack surface was assumed. Here, $F_{\mathrm{I}}$ and $F_{\mathrm{II}}$ are the normalized SIFs; for $F_{\mathrm{I}}>0$, the failure mechanism is tensile fracture, and for $F_{\mathrm{II}}>0$, the crack is 
TABLE 1: Comparison of SIFs (horizontal crack).

\begin{tabular}{|c|c|c|c|c|c|c|}
\hline$G_{(2)} / G_{(1)}$ & $d / 2 a$ & Proposed approach & Isida and Noguchi [16] & Diff. (\%) & Ryoji and Sang-Bong [17] & Diff. (\%) \\
\hline & & \multicolumn{5}{|c|}{$K_{\mathrm{I}} /(p \sqrt{\pi a})$ of tip A (or B) } \\
\hline 0.25 & 0.05 & 1.476 & 1.468 & -0.57 & 1.468 & -0.54 \\
\hline 0.25 & 0.5 & 1.198 & 1.197 & -0.09 & 1.197 & -0.12 \\
\hline 2.0 & 0.05 & 0.871 & 0.872 & 0.14 & 0.869 & -0.17 \\
\hline \multirow[t]{2}{*}{2.0} & 0.5 & 0.936 & 0.935 & -0.06 & 0.934 & -0.16 \\
\hline & & \multicolumn{5}{|c|}{$K_{\mathrm{II}} /(p \sqrt{\pi a})$ of tip A (or B) } \\
\hline 0.25 & 0.05 & 0.285 & 0.286 & 0.35 & 0.292 & 2.50 \\
\hline 0.25 & 0.5 & 0.071 & 0.071 & 0.70 & 0.072 & 1.67 \\
\hline 2.0 & 0.05 & -0.088 & -0.087 & -1.38 & -0.085 & -4.01 \\
\hline 2.0 & 0.5 & -0.023 & -0.024 & 2.50 & -0.023 & -3.54 \\
\hline
\end{tabular}

TABLE 2: Elastic properties for materials \#1 and \#2.

\begin{tabular}{lcccc}
\hline Material & $E(\mathrm{MPa})$ & $E^{\prime}(\mathrm{MPa})$ & $\nu^{\prime}$ & $G^{\prime}$ \\
\hline Material \#1 & 100 & 50 & 0.3 & 10.009 \\
Material \#2 & & & & \\
(i) & 100 & 45 & 0.3 & 9.525 \\
(ii) & 100 & 40 & 0.3 & 9.010 \\
(iii) & 100 & 30 & 0.3 & 7.860 \\
(iv) & 100 & 10 & 0.3 & 4.630 \\
\hline
\end{tabular}

subjected to an anticlockwise shear force. For a 2D problem under mixed mode loading, the failure mechanism may be defined by the SIFs of crack tips (tensile fracture, $F_{\mathrm{I}} / F_{\mathrm{II}}>1$, and shear fracture, $F_{\mathrm{I}} / F_{\mathrm{II}}<1$, resp.).

4.1. Effect of the Degree of Anisotropy on the Stress Intensity Factor. In this chapter, we analyze the SIFs for an anisotropic bi-material with various anisotropic directions, $\psi$, and degrees of material anisotropy using the BEM program. The normalized SIFs, defined as $F_{\mathrm{I}}$ and $F_{\mathrm{II}}$, are equal to

$$
F_{\mathrm{I}}=\frac{K_{\mathrm{I}}}{K_{0}}, \quad F_{\mathrm{II}}=\frac{K_{\mathrm{II}}}{K_{0}},
$$

with

$$
K_{0}=\sigma \sqrt{\pi a}
$$

In order to evaluate the influence of bi-material anisotropy on the SIFs, we considered the following three cases: (i) a horizontal crack within material \#1, (ii) a horizontal crack within material \#2, and (iii) a crack at the interface of the materials. The infinite bi-material was subjected to farfield vertical tensile stresses, as shown in Figure 5. The crack, which had a length of $2 a$, was located at distances $d / a=1$ (material \#1), -1 (material \#2), and $d / a=0$ (interfacial crack) from the interface, respectively. Material \#1 was transversely isotropic with the plane of transverse isotropy inclined at angle $\psi_{\# 1}$ with respect to the $x$-axis. Material \#1 had five independent elastic constants $\left(E_{\# 1}, E_{\# 1}^{\prime}, v_{\# 1}, v_{\# 1}^{\prime}\right.$, and $\left.G_{\# 1}\right)$ in the local coordinate system; $E_{\# i}$ and $E_{\# i}^{\prime}$ are the Young's moduli in the plane of transverse isotropy and in a direction

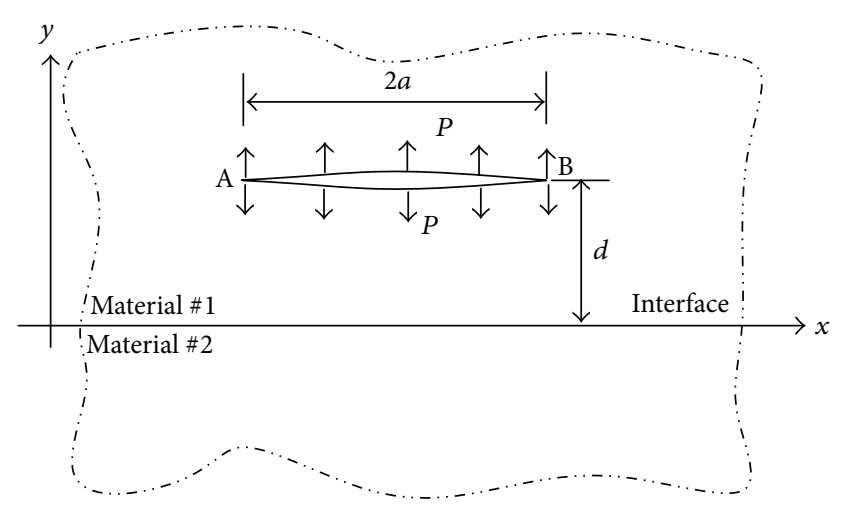

FIGURE 3: Horizontal crack under uniform pressure in material \#1 of an infinite bi-material.

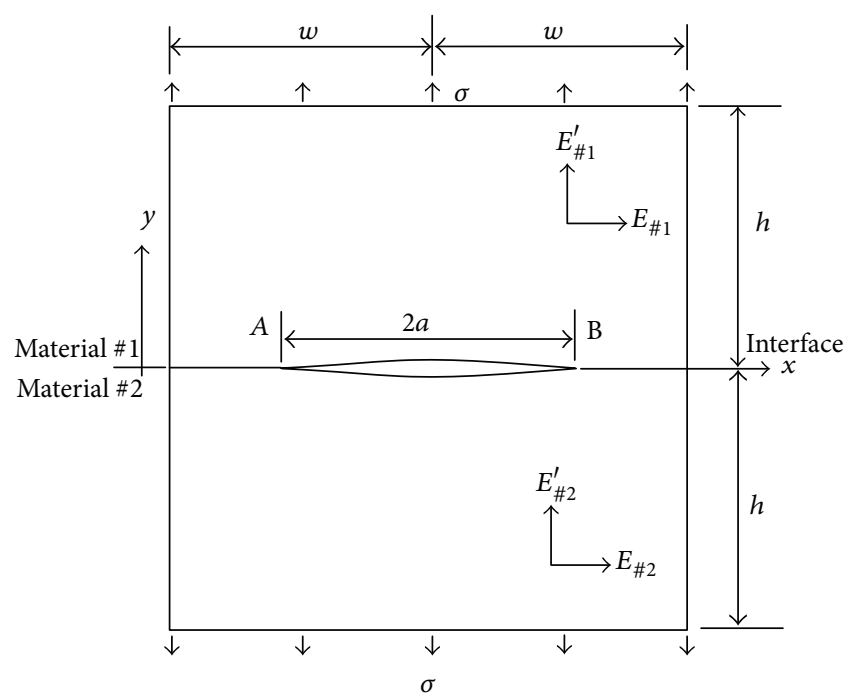

FIGURE 4: Interfacial crack within a finite rectangular plate of a bimaterial.

normal to it, respectively; $v_{\# i}$ and $\nu_{\# i}^{\prime}$ are the Poisson's ratios that characterize the lateral strain response in the plane of transverse isotropy to a stress acting parallel and normal to it, respectively; and $G_{\# i}^{\prime}$ is the shear modulus in the planes 
TABLE 3: Comparison of the normalized complex SIFs for finite anisotropic problem (interfacial crack).

\begin{tabular}{|c|c|c|c|c|c|}
\hline \multirow{2}{*}{$\begin{array}{l}\text { Material \#2 } \\
E_{\# 2}^{\prime} / E_{\# 2}\end{array}$} & \multicolumn{5}{|c|}{$|K| /(\sigma \sqrt{\pi a})$ of tip $\mathrm{A}($ or B $)$} \\
\hline & Sang-Bong et al. [18] & Wünsche et al. [8] & Diff. (\%) & Proposed approach & Diff. (\%) \\
\hline (i) 0.45 & 1.317 & 1.312 & 0.38 & 1.3132 & 0.29 \\
\hline (ii) 0.40 & 1.337 & 1.333 & 0.30 & 1.3351 & 0.14 \\
\hline (iii) 0.30 & 1.392 & 1.386 & 0.43 & 1.3922 & -0.01 \\
\hline (iv) 0.10 & 1.697 & 1.689 & 0.47 & 1.6968 & -0.06 \\
\hline
\end{tabular}

$$
{ }^{*}|K|=\sqrt{K_{\mathrm{I}}^{2}+K_{\mathrm{II}}^{2}} \text {. }
$$

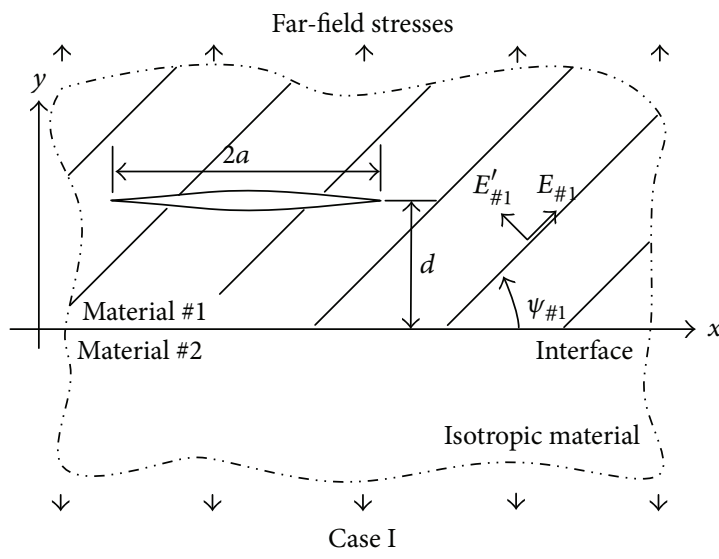

(a) Horizontal crack within material \#1

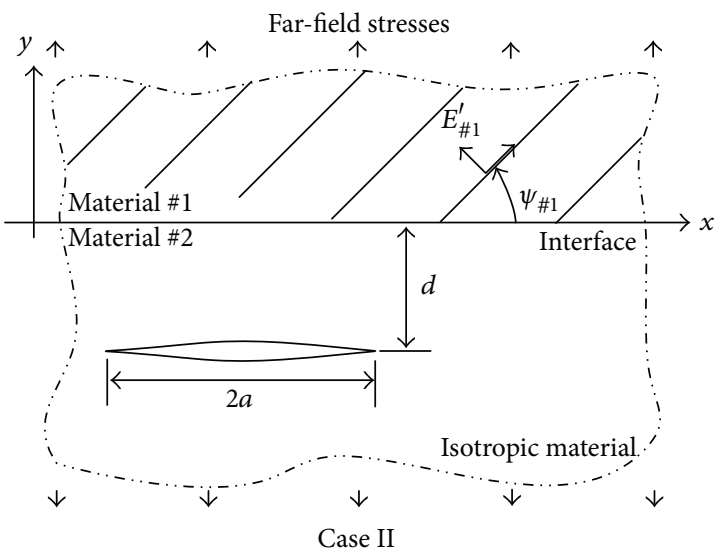

(b) Horizontal crack within material \#2

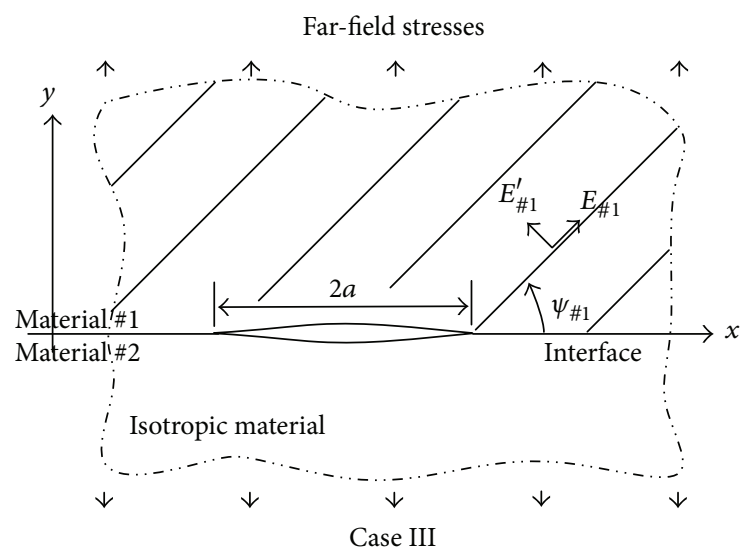

(c) Interfacial crack within bi-material

FIGURE 5: Horizontal cracks in an infinite bi-material under far-field stresses.

normal to the plane of transverse isotropy. For material \#2, $E_{\# 2} / E_{\# 2}^{\prime}=1, E_{\# 2} / G_{\# 2}^{\prime}=2.5$, and $v_{\# 2}=v_{\# 2}^{\prime}=0.25$. In this problem, the anisotropic direction $(\psi)$ within material \#1 varies from $0^{\circ}$ to $180^{\circ}$.

For all cases, three sets of dimensionless elastic constants are considered. They are defined in Table 4 . Here, $E_{\# 1} / E_{\# 2}$, which is the ratio of the Young's modulus of material \#1 to the Young's modulus of material \#2, equals one. In order to calculate the SIFs at the crack tip, 20 quadratic elements were used to discretize the crack surface. The numerical results are plotted in Figures 7 to 9 for cases I, II, and III, respectively. The results for the isotropic case $\left(E_{\# 1} / E_{\# 1}^{\prime}=1, E_{\# 1} / G_{\# 1}^{\prime}=2.5\right.$,
TABLE 4: Sets of dimensionless elastic constants $\left(\nu_{\# 1}=0.25\right)$.

\begin{tabular}{lcc}
\hline$E_{\# 1} / E_{\# 1}^{\prime}$ & $E_{\# 1} / G_{\# 1}^{\prime}$ & $\nu_{\# 1}^{\prime}$ \\
\hline $1 / 3,1 / 2,1,2,3$ & 2.5 & 0.25 \\
\hline${ }^{*}$ Subscript \#1 is applied in material \#1. &
\end{tabular}

and $\left.v_{\# 1}^{\prime}=0.25\right)$ are shown as solid lines in the figures for comparison.

4.2. An Inclined Crack Situated Near the Interfacial Crack. This case treats interaction between the interfacial crack 


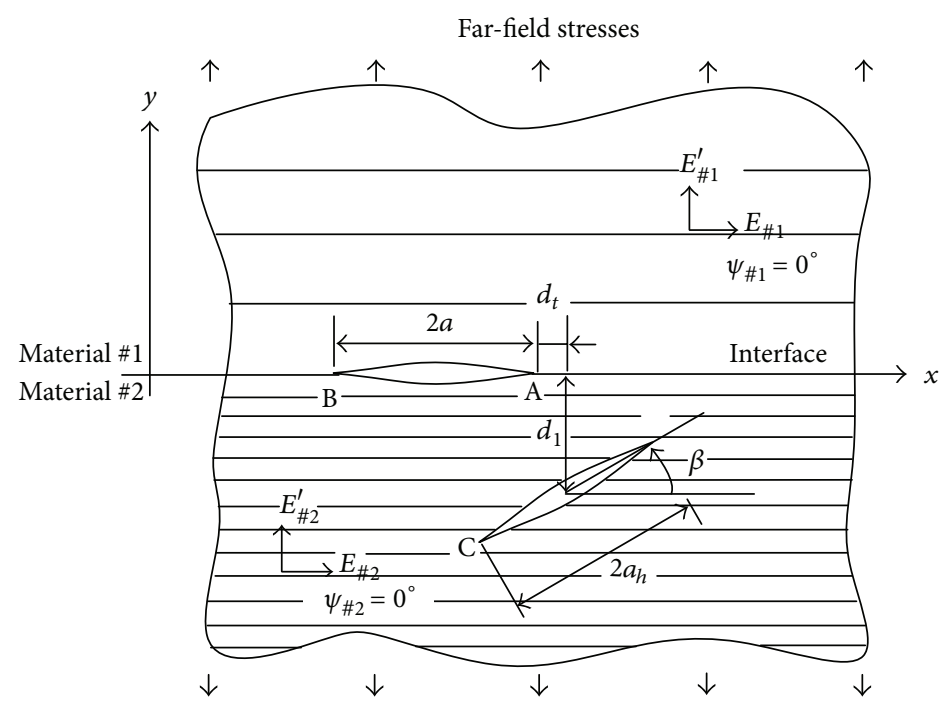

FIGURE 6: A crack situated near the interfacial crack within infinite bi-materials.

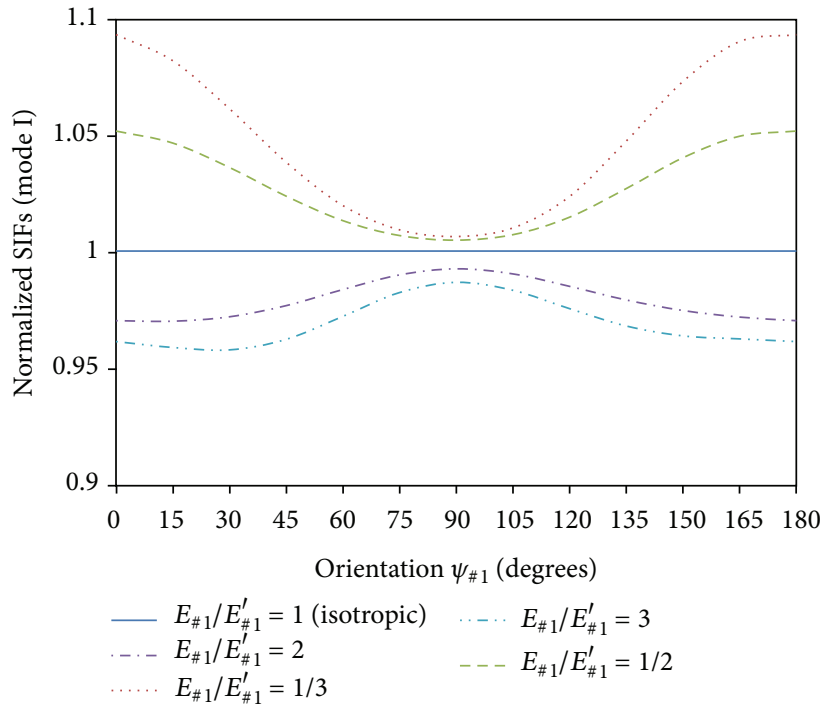

(a)

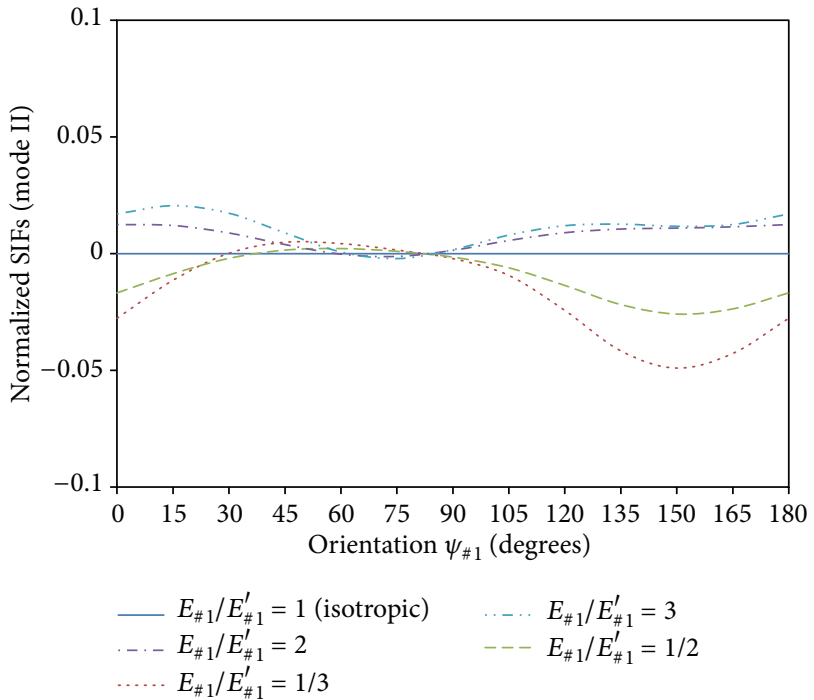

(b)

FIGURE 7: Variation of normalized SIFs of the crack tip in material \#1 with the anisotropic directions $\psi_{\# 1}$ for case I $\left(E_{\# 1} / G_{\# 1}^{\prime}=2.5, v_{\# 1}^{\prime}=\right.$ $\left.0.25, E_{\# 1} / E_{\# 2}=1\right)$.

and an inclined crack with an angle $\beta$. The ratio $d_{t} / a=$ 0.25 , respectively. The same consider an infinite plate of bimaterials, having the elastic constants ratio $E_{\# 1} / E_{\# 1}^{\prime}=1$, $E_{\# 1} / G_{\# 1}^{\prime}=0.4$, and $v_{\# 1}^{\prime}=0.2499$ for material \#1 and $E_{\# 2} / E_{\# 2}^{\prime}=1 / 3, E_{\# 2} / G_{\# 2}^{\prime}=0.4$, and $v_{\# 2}^{\prime}=0.2499$ for material $\# 2$. The Poisson ratios are $v_{\# 1}=v_{\# 2}=0.25$, material orientation $\psi_{\# 1}=\psi_{\# 2}=0^{\circ}$, and the Young modulus ratio $E_{\# 1} / E_{\# 2}=$ $1 / 5$. One considers an interfacial crack of length $2 a$ and a horizontal crack situated near the interfacial crack with ah. The ratio $a / a_{h}$ is equal to 1 . The longitudinal distance $d_{l} / a=1$, and the plate is subjected to a far-field vertical tensile stresses as shown in Figure 6. For each crack, surface is meshed by 20 quadratic elements. The plane stress conditions were supposed. Calculations were carried out by our BEM code.
Figures 10 and 11 represent the variations of normalized SIFs $\left(F_{\mathrm{I}}\right.$ and $\left.F_{\mathrm{II}}\right)$ of interfacial crack and inclined crack according to the inclined angle $\beta$, respectively.

4.3. Discussion. An analysis of Figures 7 to 9 reveals the following major trends.

(i) For the SIFs in mode I, the orientation of the planes of material anisotropy with respect to the horizontal plane has a strong influence on the value of the SIFs for case I and case II. The influence is small for case III. This means that the effects of mode I on the crack in the homogeneous material are greater than those of the interface. 


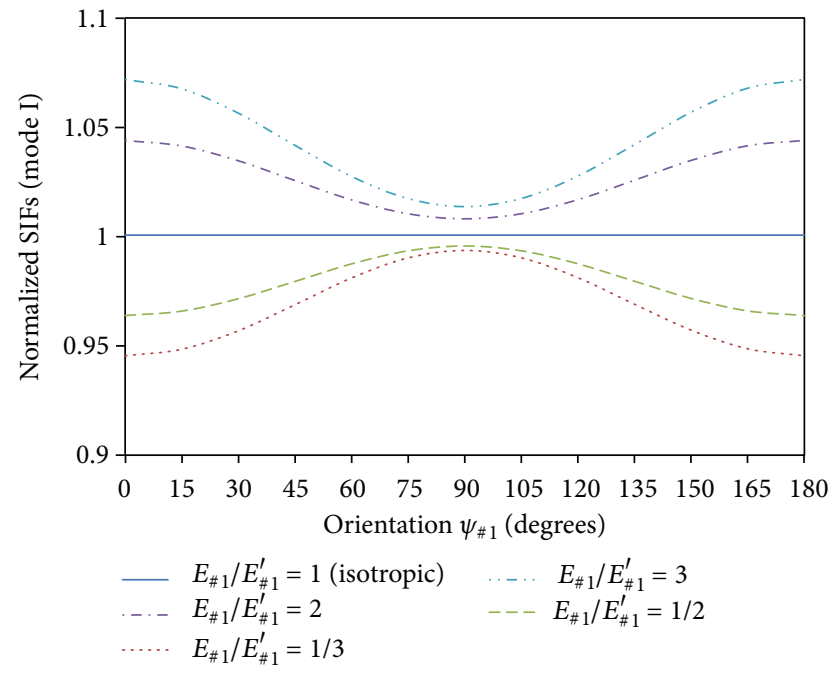

(a)

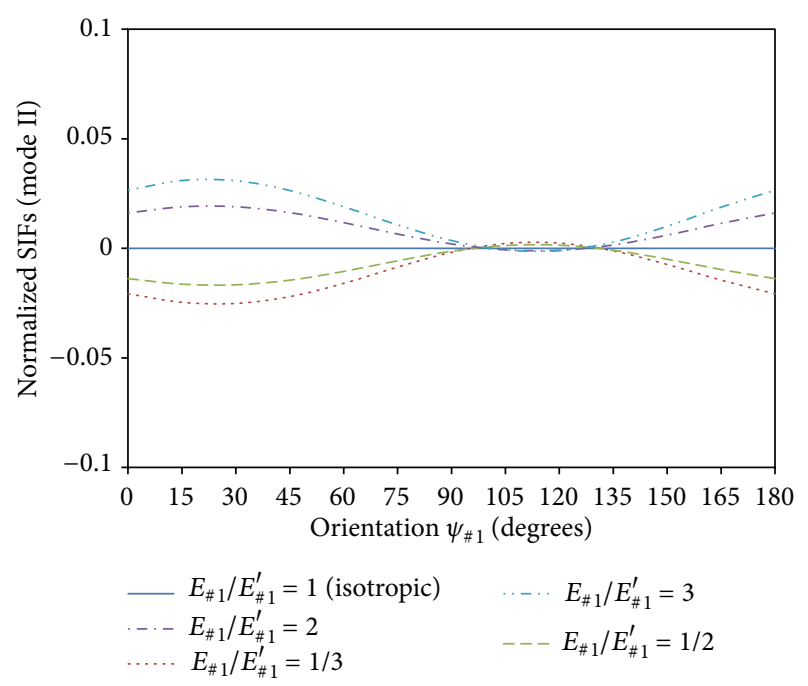

(b)

FIGURE 8: Variation of Normalized SIFs of crack tip in material \#2 with the anisotropic directions $\psi_{\# 1}$ for case II $\left(E_{\# 1} / G_{\# 1}^{\prime}=2.5, v_{\# 1}^{\prime}=\right.$ $\left.0.25, E_{\# 1} / E_{\# 2}=1\right)$.

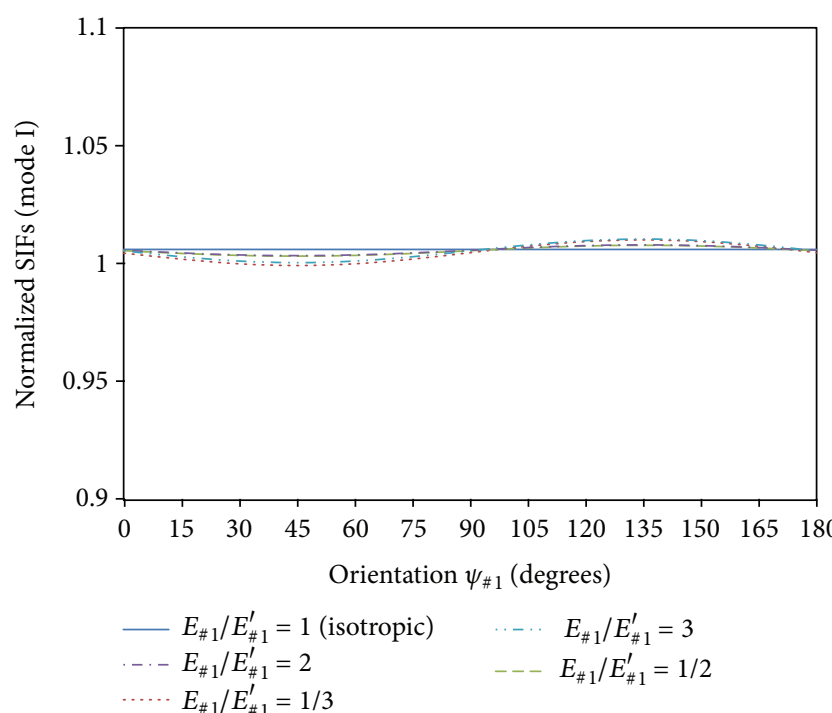

(a)

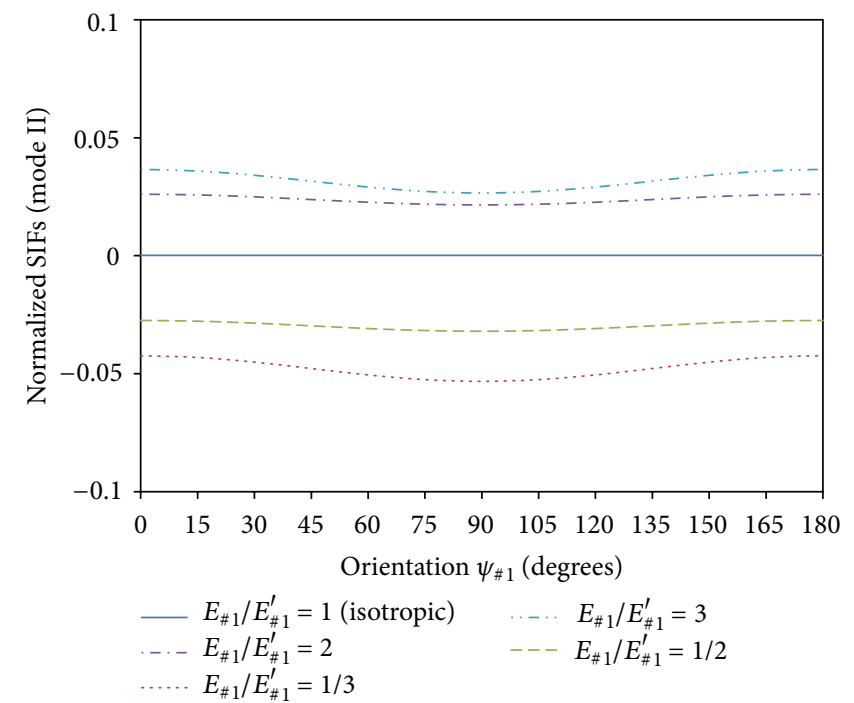

(b)

FIGURE 9: Variation of normalized SIFs of interfacial crack tip with the anisotropic directions $\psi_{\# 1}$ for case III $\left(E_{\# 1} / G_{\# 1}^{\prime}=2.5, v_{\# 1}^{\prime}=\right.$ $\left.0.25, E_{\# 1} / E_{\# 2}=1\right)$.

(ii) For all cases, the variation of the SIFs with the anisotropic direction $\psi_{\# 1}$ is symmetric with respect to $\psi_{\# 1}=90^{\circ}$. However, this type of symmetry was not observed for the SIFs (mode II) in case 1 and case 2 (Figures 7(b) and 8(b)).

(iii) For the interfacial crack (case III), the anisotropic direction $\psi_{\# 1}$ has a strong influence on the value of the SIFs for mode II (Figure 9(b)). The influence is small for mode I. It should be noted that the effects of $E_{\# 1} / E_{\# 1}^{\prime}$ on the SIFs of mode II are greater than those of mode I. (iv) There is a greater variation in the SIFs with the anisotropic direction $\psi_{\# 1}$ for bi-materials with a high degree of anisotropy $\left(E_{\# 1} / E_{\# 1}^{\prime}=3\right.$ or $\left.1 / 3\right)$.

(v) Figure $7(\mathrm{a})\left(E_{* 1} / E_{* 1}^{\prime}=1 / 3\right)$ indicates that the maximum values of the SIFs (mode I) occur when the far-field stresses are perpendicular to the plane of transverse isotropy (i.e., $\psi_{\# 1}=0^{\circ}$ or $180^{\circ}$ ) for case 1 (crack within material \#1). Figure 9 (b) $\left(E_{\# 1} / E_{\# 1}^{\prime}=\right.$ $1 / 3$ ) shows that the minimum values of the SIFs (mode II) occur when the anisotropic direction angle is about $90^{\circ}$ for case 3 (interfacial crack). 


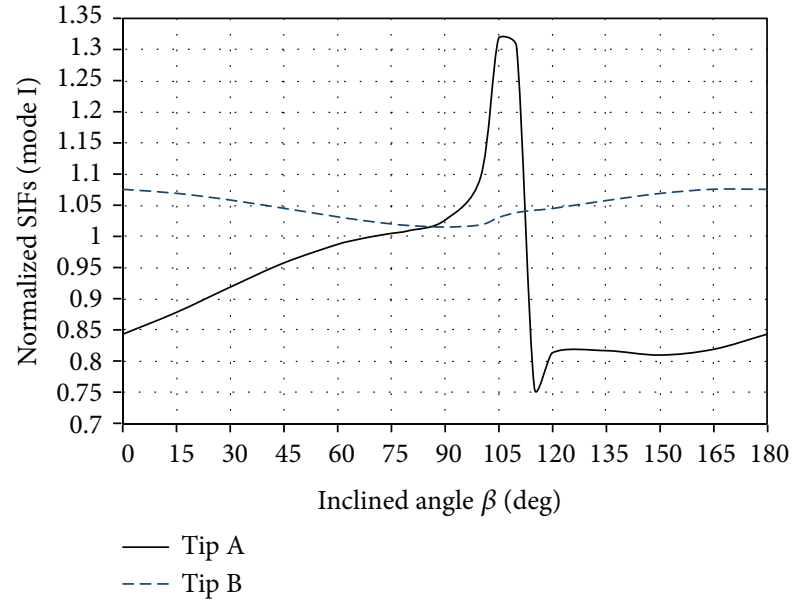

(a)

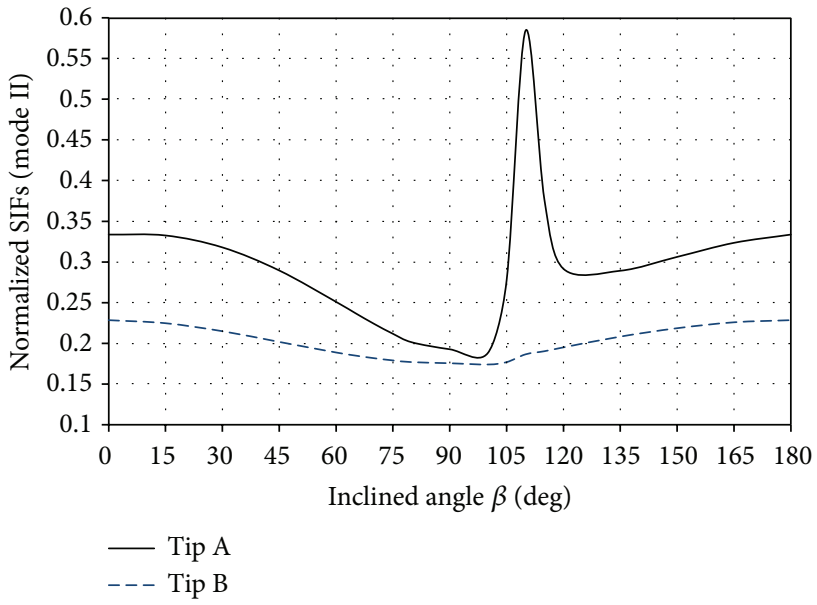

(b)

FIGURE 10: Variation of normalized SIFs of an interfacial crack with an inclined crack angle $\beta$.

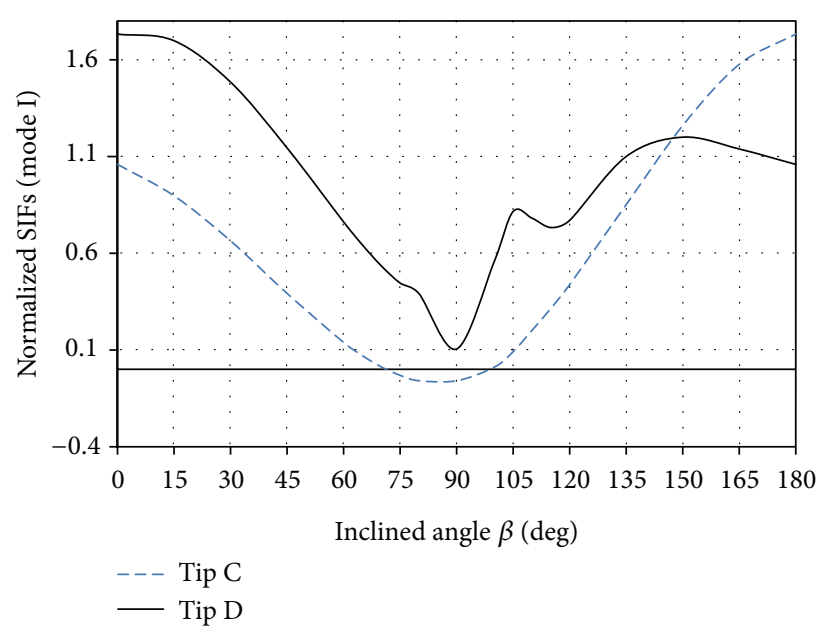

(a)

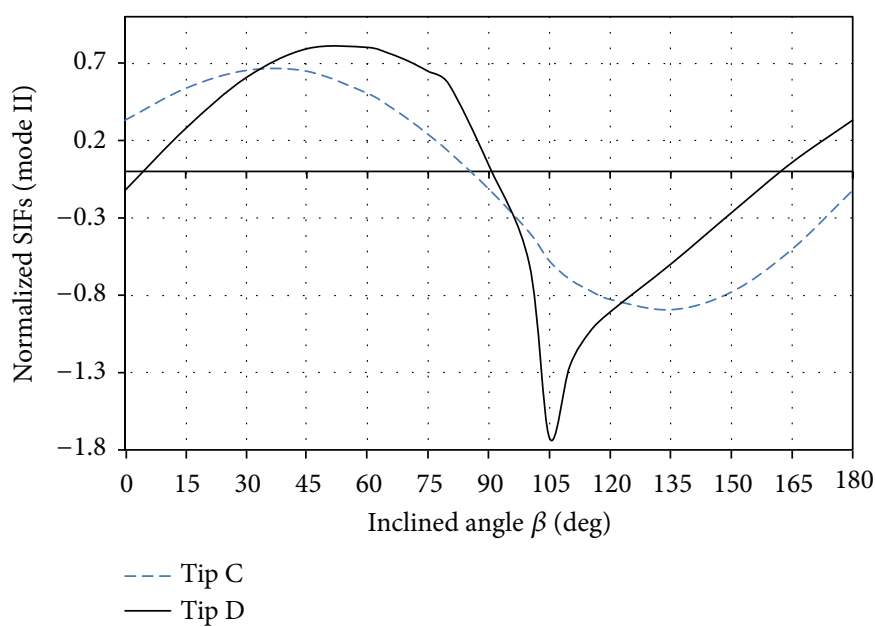

(b)

FIGURE 11: Variation of normalized SIFs of an inclined crack with the inclined angle $\beta$.

Analysis of Figures 10 and 11 reveals that several major trends can be underlined as follows.

(vi) Figure 10(a) presents the SIFs (mode I) variation as an inclined angle $\beta$ of the inclined crack for different values of the interfacial crack. It is shown that the increase in the inclined angle $\beta$ causes a slight increase in SIFs (mode I) until reaching a value at $\beta=90^{\circ}$. From these angles, the tip D meets at the interface of bi-materials and the normalized SIFs start an immediate sharp increasing. The rise continued until it reached $\beta=105^{\circ}$. The maximum of SIFs (mode I) is reached for this last angle. In this position, the distance between tip D and the interfacial crack is minimal which increases the stress interaction between the two crack tips. For $\beta>120^{\circ}$, the SIFs start decreasing and stabilizing when approaching $180^{\circ}$. (vii) The same phenomenon was observed in Figure 10(b). It is shown that the increase in the inclined angle $\beta$ causes the reduction in SIFs (mode II) until reaching a value at $\beta=100^{\circ}$, and starts an immediate sharp increasing. The maximum of SIFs (mode II) is reached for $\beta=110^{\circ}$. In this position, the distance between tip $\mathrm{D}$ and the interfacial crack is minimal which increases the stress interaction between the two crack tips. For $\beta>120^{\circ}$, the SIFs start a slight increasing and stabilizing when approaching $180^{\circ}$.

(viii) Figure 11 illustrates, respectively, the variations of the normalized SIFs of tip C and D according to inclined angle $\beta$. For Figure 11(a), between $0^{\circ}$ and $90^{\circ}$, one observes that mode I of the normalized SIFs decreases with $\beta$. The minimal value is reached at $\beta=90^{\circ}$. From $90^{\circ}$, the two normalized SIFs increase until $138^{\circ}$, and the normalized SIFs at the tip D reaches a maximum 


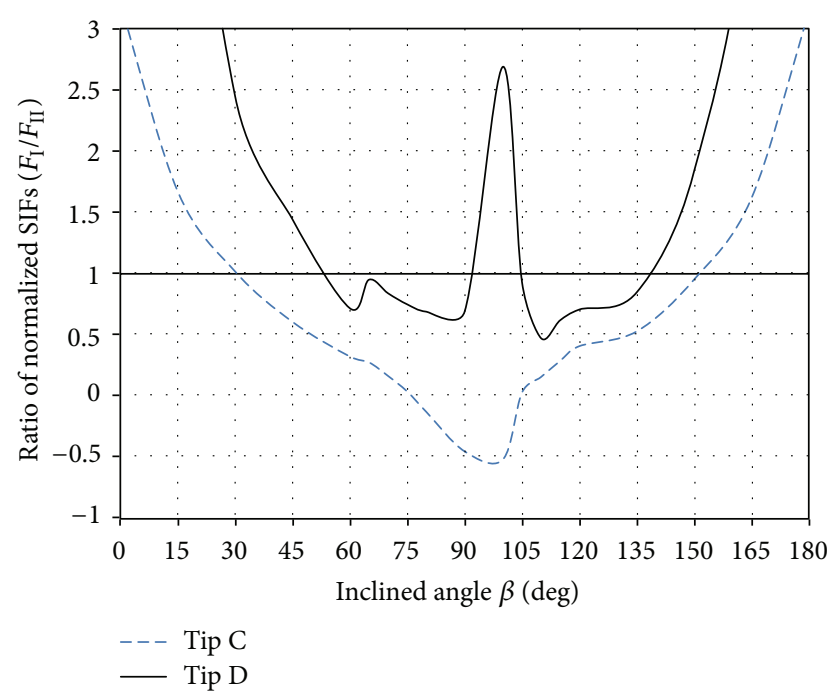

FIGURE 12: Variation of the ratio of $F_{\mathrm{I}} / F_{\mathrm{II}}$ of an inclined crack with the inclined angle $\beta$.

value. In the interval of $90^{\circ}$ at $138^{\circ}$, the normalized SIFs of tip D are higher than those of tip C, because the tip D approaches the tip of the interfacial crack. Between $138^{\circ}$ and $180^{\circ}$, tip D moves away from the tip A of interfacial crack.

(ix) For Figure 11(b), the normalized SIFs (mode II) of the two tips grow in interval of the angles ranging between $0^{\circ}$ and $35^{\circ}$ and decrease between $35^{\circ}$ and $80^{\circ}$. In the interval of $0^{\circ}$ at $32^{\circ}$ the normalized SIFs of tip C are slightly higher than those of tip D, and the inverse phenomenon is noted for angles ranging between $32^{\circ}$, and $100^{\circ}$. These results permit to note on one hand that the orientation of the inclined crack has influence on the variations of $F_{\mathrm{I}}$ and a weak influence on the variations of $F_{\mathrm{II}}$, and in another hand, when the angle of this orientation increases, the normalized SIFs of mode I increase and automatically generate the reduction in mode II SIFs. For homogeneous and isotropic materials, the two SIFs for inclined crack are nulls at $\beta=90^{\circ}$. The presence of the bi-material interface gives $F_{\mathrm{I}}$ different of zero whatever the value of $\beta$ and $F_{\mathrm{II}}=0$ at $\beta=80^{\circ}$.

(x) The ratio of the normalized SIFs $\left(F_{\mathrm{I}} / F_{\mathrm{II}}\right)$ is concerned; Figure 12 shows that the fracture of shear mode $\left(F_{\mathrm{I}} / F_{\mathrm{II}}<1\right)$ occurs to the inclined crack angle is between $30^{\circ}$ and $150^{\circ}$ (tip C). For tip D, the shear mode $\left(F_{\mathrm{I}} / F_{\mathrm{II}}<1\right)$ occurs to the $\beta$ is between $50^{\circ}$ and $95^{\circ}$, and between $105^{\circ}$ and $120^{\circ}$, respectively. And the opening mode $\left(F_{\mathrm{I}} / F_{\mathrm{II}}>1\right)$ occurs to the $\beta$ is between $95^{\circ}$ and $105^{\circ}$. In this position, the distance between tip $\mathrm{D}$ and tip $\mathrm{A}$ of the interfacial crack is minimal which increases the stress interaction between the two crack tips.

\section{Conclusions}

In this study, we developed a single-domain BEM formulation in which neither the artificial boundary nor the discretization along the uncracked interface is necessary. Fedeliński [11] presented the single-domain BEM formulation for homogeneous materials. We combined Chen's formulation with the Green's functions of bi-materials derived by Ke et al. [12] to extend it to anisotropic bi-materials. The major achievements of the research work are summarized in the following.

(i) A decoupling technique was used to determine the SIFs of the mixed mode and the oscillation on the interfacial crack based on the relative displacements at the crack tip. Five types of three-node quadratic elements were utilized to approximate the crack tip and the outer boundary. A crack surface and an interfacial crack surface were evaluated using the asymptotical relation between the SIFs. Since the interfacial crack has an oscillation singular behavior, we used a special crack-tip element [26] to capture this behavior.

(ii) Calculation of the SIFs was conducted for several situations, including cracks along and away from the interface. Numerical results show that the proposed method is very accurate, even with relatively coarse mesh discretization. In addition, the use of proposed BEM program is also very fast. The calculation time for each sample was typically $10 \mathrm{~s}$ on a PC with an Intel Core i7-2600 CPU at $3.4 \mathrm{GHz}$ and $4 \mathrm{~GB}$ of RAM.

(iii) The study of the fracture behavior of a crack is of practical importance due to the increasing application of anisotropic bi-material. Therefore, the fracture mechanisms for anisotropic bi-material need to be investigated.

\section{Acknowledgments}

The authors would like to thank the National Science Council of the Republic of China, Taiwan, for financially supporting this research under Contract no. NSC-100-2221-E-006220-MY3. They also would like to thank two anonymous reviewers for their very constructive comments that greatly improved the paper.

\section{References}

[1] M. L. Williams, "The stresses around a fault or crack in dissimilar media," Bulletin of the Seismological Society of America, vol. 49, pp. 199-204, 1959.

[2] A. H. England, "A crack between dissimilar media," Journal of Applied Mechanics, vol. 32, pp. 400-402, 1965.

[3] J. R. Rice and G. C. Sih, "Plane problems of cracks in dissimilar media," Journal of Applied Mechanics, vol. 32, pp. 418-423, 1965.

[4] J. R. Rice, "Elastic fracture mechanics concepts for interfacial crack," Journal of Applied Mechanics, vol. 55, pp. 98-103, 1988. 
[5] D. L. Clements, "A crack between dissimilar anisotropic media," International Journal of Engineering Science, vol. 9, pp. 257-265, 1971.

[6] J. R. Willis, "Fracture mechanics of interfacial cracks," Journal of the Mechanics and Physics of Solids, vol. 19, no. 6, pp. 353-368, 1971.

[7] K. C. Wu, "Stress intensity factor and energy release rate for interfacial cracks between dissimilar anisotropic materials," Journal of Applied Mechanics, vol. 57, pp. 882-886, 1990.

[8] M. Wünsche, C. Zhang, J. Sladek, V. Sladek, S. Hirose, and M. Kuna, "Transient dynamic analysis of interface cracks in layered anisotropic solids under impact loading," International Journal of Fracture, vol. 157, no. 1-2, pp. 131-147, 2009.

[9] J. Y. Jhan, C. S. Chen, C. H. Tu, and C. C. Ke, "Fracture mechanics analysis of interfacial crack in anisotropic bi-materials," Key Engineering Materials, vol. 467-469, pp. 1044-1049, 2011.

[10] J. Lei and C. Zhang, "Time-domain BEM for transient interfacial crack problems in anisotropic piezoelectric bi-materials," International Journal of Fracture, vol. 174, pp. 163-175, 2012.

[11] P. Fedeliński, "Computer modelling and analysis of microstructures with fibres and cracks," Journal of Achievements in Materials and Manufacturing Engineering, vol. 54, no. 2, pp. 242-249, 2012.

[12] C. C. Ke, C. L. Kuo, S. M. Hsu, S. C. Liu, and C. S. Chen, "Two-dimensional fracture mechanics analysis using a singledomain boundary element method," Mathematical Problems in Engineering, vol. 2012, Article ID 581493, 26 pages, 2012.

[13] J. Xiao, W. Ye, Y. Cai, and J. Zhang, "Precorrected FFT accelerated BEM for large-scale transient elastodynamic analysis using frequency-domain approach," International Journal for Numerical Methods in Engineering, vol. 90, no. 1, pp. 116-134, 2012.

[14] C. S. Chen, E. Pan, and B. Amadei, "Fracture mechanics analysis of cracked discs of anisotropic rock using the boundary element method," International Journal of Rock Mechanics and Mining Sciences, vol. 35, no. 2, pp. 195-218, 1998.

[15] E. Pan and B. Amadei, "Boundary element analysis of fracture mechanics in anisotropic bimaterials," Engineering Analysis with Boundary Elements, vol. 23, no. 8, pp. 683-691, 1999.

[16] M. Isida and H. Noguchi, "Plane elastostatic problems of bonded dissimilar materials with an interface crack and arbitrarily located cracks," Transactions of the Japan Society of Mechanical Engineering, vol. 49, pp. 137-146, 1983 (Japanese).

[17] Y. Ryoji and C. Sang-Bong, "Efficient boundary element analysis of stress intensity factors for interface cracks in dissimilar materials," Engineering Fracture Mechanics, vol. 34, no. 1, pp. 179-188, 1989.

[18] C. Sang-Bong, L. R. Kab, C. S. Yong, and Y. Ryoji, "Determination of stress intensity factors and boundary element analysis for interface cracks in dissimilar anisotropic materials," Engineering Fracture Mechanics, vol. 43, no. 4, pp. 603-614, 1992.

[19] S. G. Lekhnitskii, Theory of Elasticity of an Anisotropic Elastic Body, Translated by P. Fern. Edited by Julius J. Brandstatter, Holden-Day, San Francisco, Calif, USA, 1963.

[20] Z. Suo, "Singularities, interfaces and cracks in dissimilar anisotropic media," Proceedings of the Royal Society. London. Series A, vol. 427, no. 1873, pp. 331-358, 1990.

[21] T. C. T. Ting, Anisotropic Elasticity, vol. 45 of Oxford Engineering Science Series, Oxford University Press, New York, NY, USA, 1996.
[22] E. Pan and B. Amadei, "Fracture mechanics analysis of cracked 2-D anisotropic media with a new formulation of the boundary element method," International Journal of Fracture, vol. 77, no. 2, pp. 161-174, 1996.

[23] P. Sollero, M. H. Aliabadi, and D. P. Rooke, "Anisotropic analysis of cracks emanating from circular holes in composite laminates using the boundary element method," Engineering Fracture Mechanics, vol. 49, no. 2, pp. 213-224, 1994.

[24] S. L. Crouch and A. M. Starfield, Boundary Element Methods in Solid Mechanics, George Allen \& Unwin, London, UK, 1983.

[25] G. Tsamasphyros and G. Dimou, "Gauss quadrature rules for finite part integrals," International Journal for Numerical Methods in Engineering, vol. 30, no. 1, pp. 13-26, 1990.

[26] E. Pan, "A general boundary element analysis of 2-D linear elastic fracture mechanics," International Journal of Fracture, vol. 88, no. 1, pp. 41-59, 1997.

[27] G. C. Sih, P. C. Paris, and G. R. Irwin, "On cracks in rectilinearly anisotropic bodies," International Journal of Fracture, vol. 3, pp. 189-203, 1965.

[28] P. Sollero and M. H. Aliabadi, "Fracture mechanics analysis of anisotropic plates by the boundary element method," International Journal of Fracture, vol. 64, no. 4, pp. 269-284, 1993.

[29] H. Gao, M. Abbudi, and D. M. Barnett, "Interfacial crack-tip field in anisotropic elastic solids," Journal of the Mechanics and Physics of Solids, vol. 40, no. 2, pp. 393-416, 1992. 


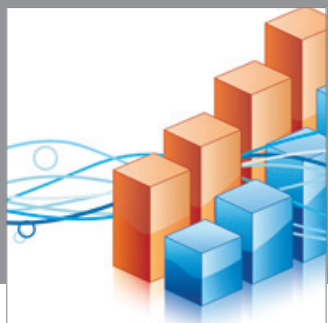

Advances in

Operations Research

mansans

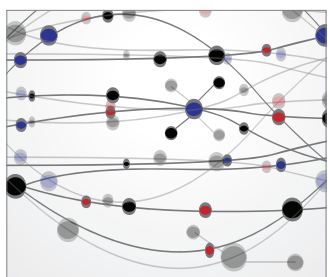

The Scientific World Journal
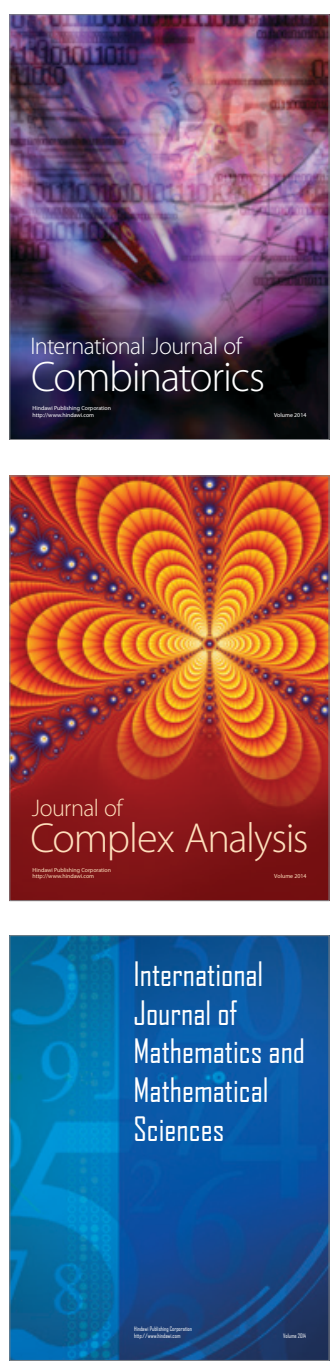
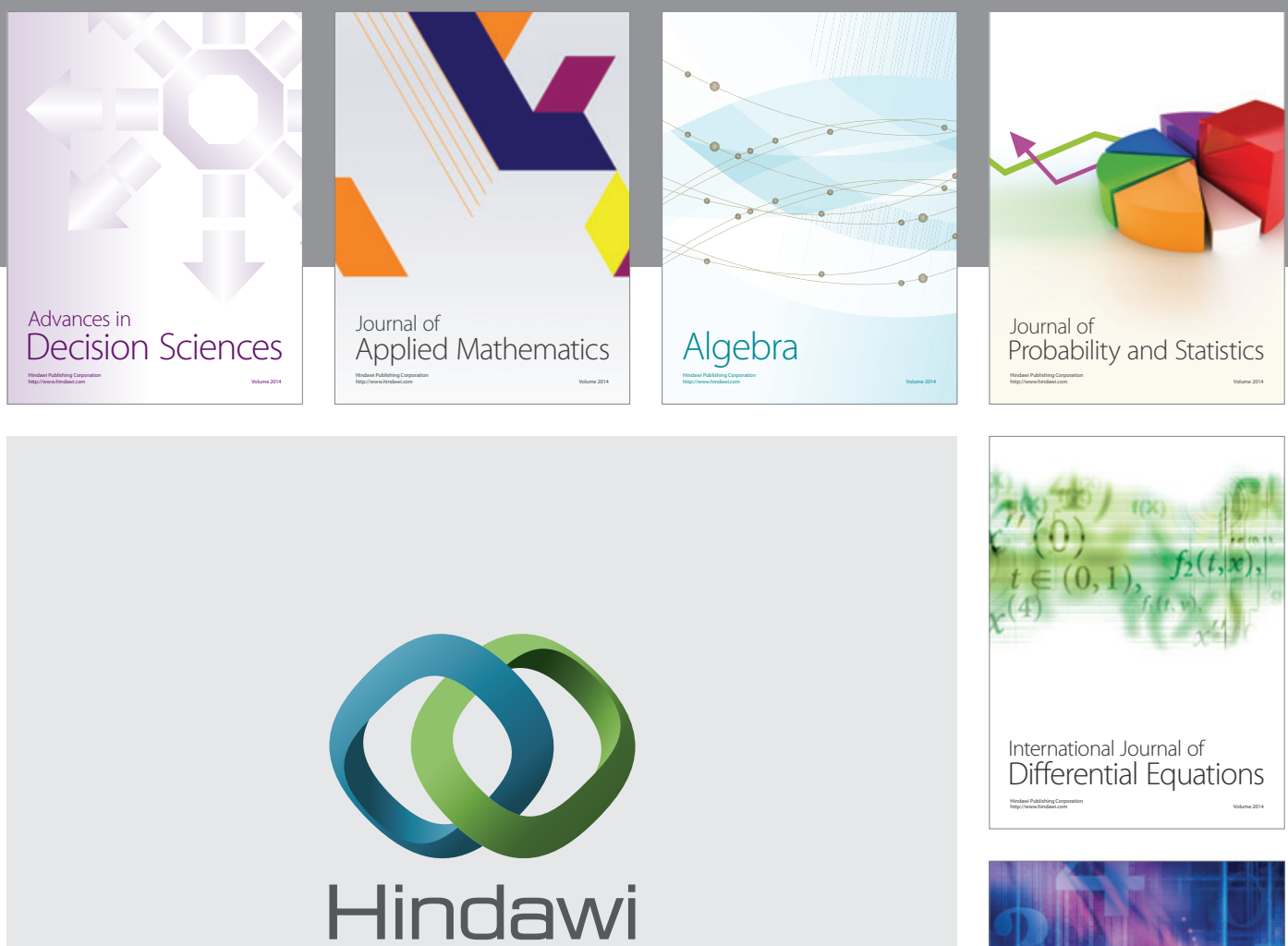

Submit your manuscripts at http://www.hindawi.com
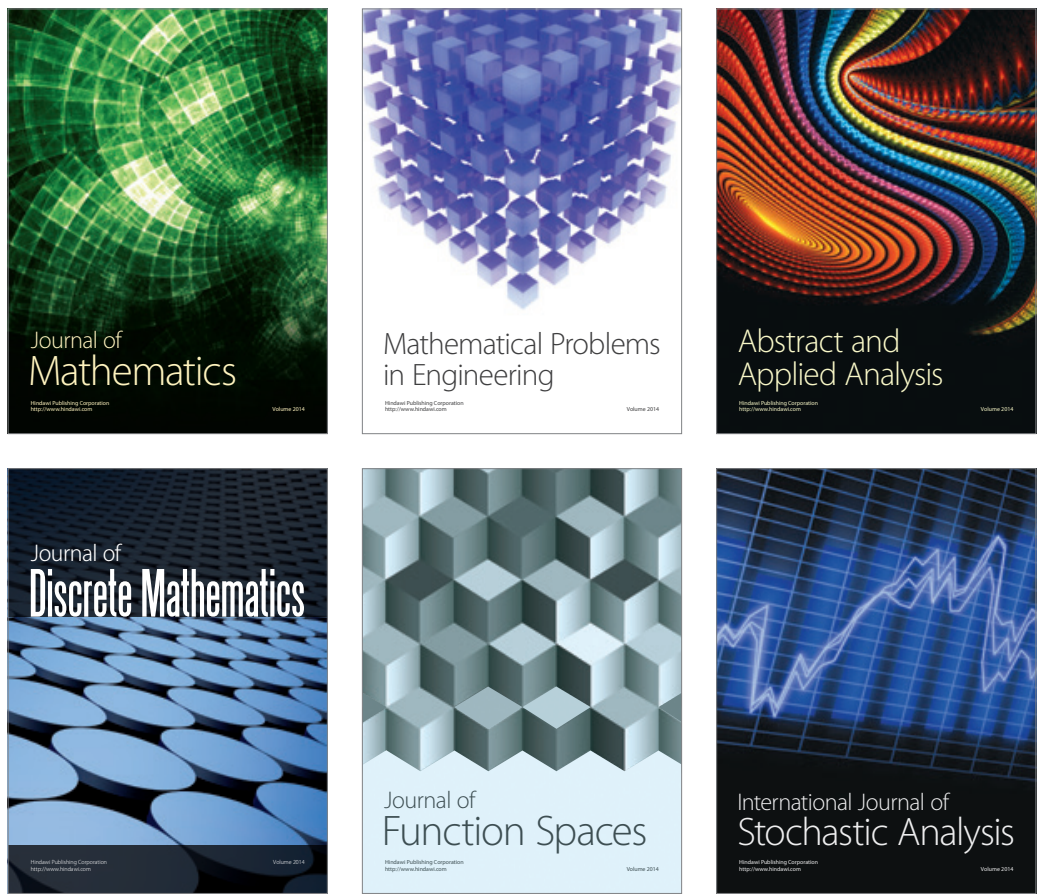

Journal of

Function Spaces

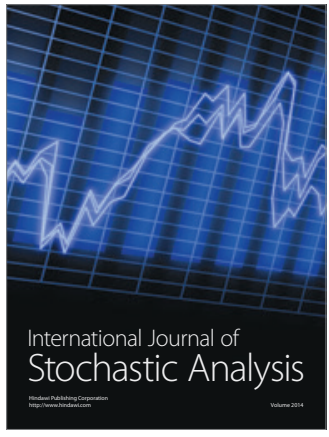

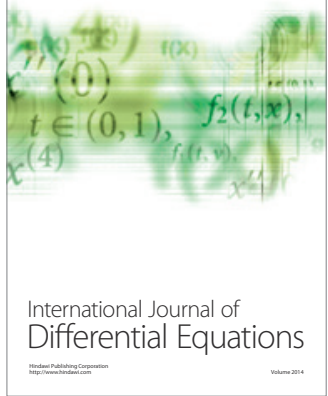
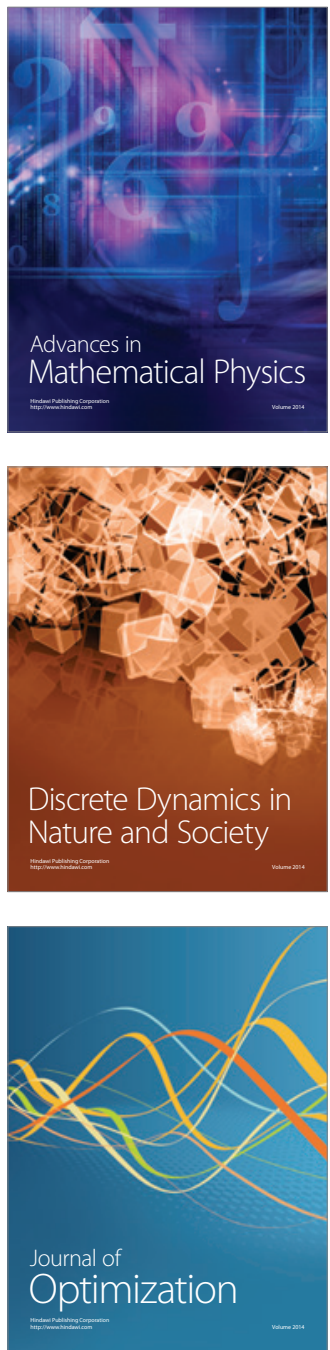